\title{
First results of electric field and density observations by Cluster EFW based on initial months of operation
}

\author{
G. Gustafsson ${ }^{1}$, M. André ${ }^{1}$, T. Carozzi ${ }^{1}$, A. I. Eriksson ${ }^{1}$, C.-G. Fälthammar ${ }^{4}$, R. Grard ${ }^{5}$, G. Holmgren ${ }^{8}$, J. A. Holtet ${ }^{3}$, \\ N. Ivchenko ${ }^{4}$, T. Karlsson ${ }^{4}$, Y. Khotyaintsev ${ }^{1}$, S. Klimov ${ }^{6}$, H. Laakso ${ }^{5}$, P.-A. Lindqvist ${ }^{4}$, B. Lybekk ${ }^{3}$, G. Marklund ${ }^{4}$, \\ F. Mozer $^{2}$, K. Mursula ${ }^{7}$, A. Pedersen ${ }^{3}$, B. Popielawska ${ }^{9}$, S. Savin ${ }^{6}$, K. Stasiewicz ${ }^{1}$, P. Tanskanen ${ }^{7}$, A. Vaivads ${ }^{1}$, and \\ J-E. Wahlund ${ }^{1}$ \\ ${ }^{1}$ Swedish Institute of Space Physics, Uppsala Division, Uppsala, Sweden \\ ${ }^{2}$ Space Sciences Laboratory, Berkeley, USA \\ ${ }^{3}$ University of Oslo, Norway \\ ${ }^{4}$ Alfvén Laboratory, Stockholm, Sweden \\ ${ }^{5}$ Space Science Department, ESTEC, Noordwijk, The Netherlands \\ ${ }^{6}$ Space Research Institute, Moscow, Russia \\ ${ }^{7}$ University of Oulu, Finland \\ ${ }^{8}$ University of Uppsala, Sweden \\ ${ }^{9}$ Space Research Centre, Warsaw, Poland
}

Received: 11 April 2001 - Revised: 3 July 2001 - Accepted: 6 July 2001

\begin{abstract}
Highlights are presented from studies of the electric field data from various regions along the CLUSTER orbit. They all point towards a very high coherence for phenomena recorded on four spacecraft that are separated by a few hundred kilometers for structures over the whole range of apparent frequencies from $1 \mathrm{mHz}$ to $9 \mathrm{kHz}$. This presents completely new opportunities to study spatialtemporal plasma phenomena from the magnetosphere out to the solar wind. A new probe environment was constructed for the CLUSTER electric field experiment that now produces data of unprecedented quality. Determination of plasma flow in the solar wind is an example of the capability of the instrument.
\end{abstract}

Key words. Magnetospheric physics (electric fields) Space plasma physics (electrostatic structures; turbulence)

\section{Introduction}

With its four-point measurements, Cluster is uniquely well adapted to study the small-scale plasma structures in space and time in key plasma regions. To fully utilize this opportunity, the Cluster Electric Field and Wave (EFW) instrument has been designed for detailed study of phenomena on spatial scales corresponding to the separation distance of the four spacecraft and their interpretation in terms of the role they play on a more global scale in shaping the properties of different regions along the orbit. Combined with the rigorous

Correspondence to: G. Gustafsson

(Georg.Gustafsson@irfu.se) pre-launch testing and calibration programme, the novel design of the probe environment enables EFW to measure electric fields with sensitivity that is unprecedented in this region of space. In this paper, we present some of the first results from EFW and show some of the unique features of this instrument. EFW data are also analysed and presented in other papers in this issue (André et al., 2001; Pedersen et al., 2001).

EFW uses two pairs of spherical probes deployed on wire booms in the spin plane to measure the electric field (Gustafsson et al., 1997). The probe-to-probe separation is $88 \mathrm{~m}$. We also use measurements of the potential of the spherical probes to the spacecraft, which is a measure of the plasma density (Pedersen et al., 1995; Pedersen et al., 2001 this issue). Data from the fluxgate magnetometer are used to support the analysis of the electric field data (Balogh et al., 1997).

To demonstrate the overall similarity between recordings from the four spacecraft, the electric field and density observations for a full orbit $(57 \mathrm{~h})$ are shown in Fig. 1. It is clear from the figure that signals from the four spacecraft look very similar on this time scale. Perigee is located at the center of the panels at 15:50 UT, 23 January 2001 and apogee is near the beginning and end of the panels. The local time of apogee was approximately 14:30 LT.

The EFW instrument has an improved design of the probe environment compared to similar instruments flown earlier. This has resulted in higher accuracy and sensitivity of the electric field measurements on Cluster. A description of the instrument is given in Sect. 2 to emphasize the advantage of the new design and introduce the various data products 
Cluster EFW 20001-01-22 11:00 -- 2001-01-24 19:00

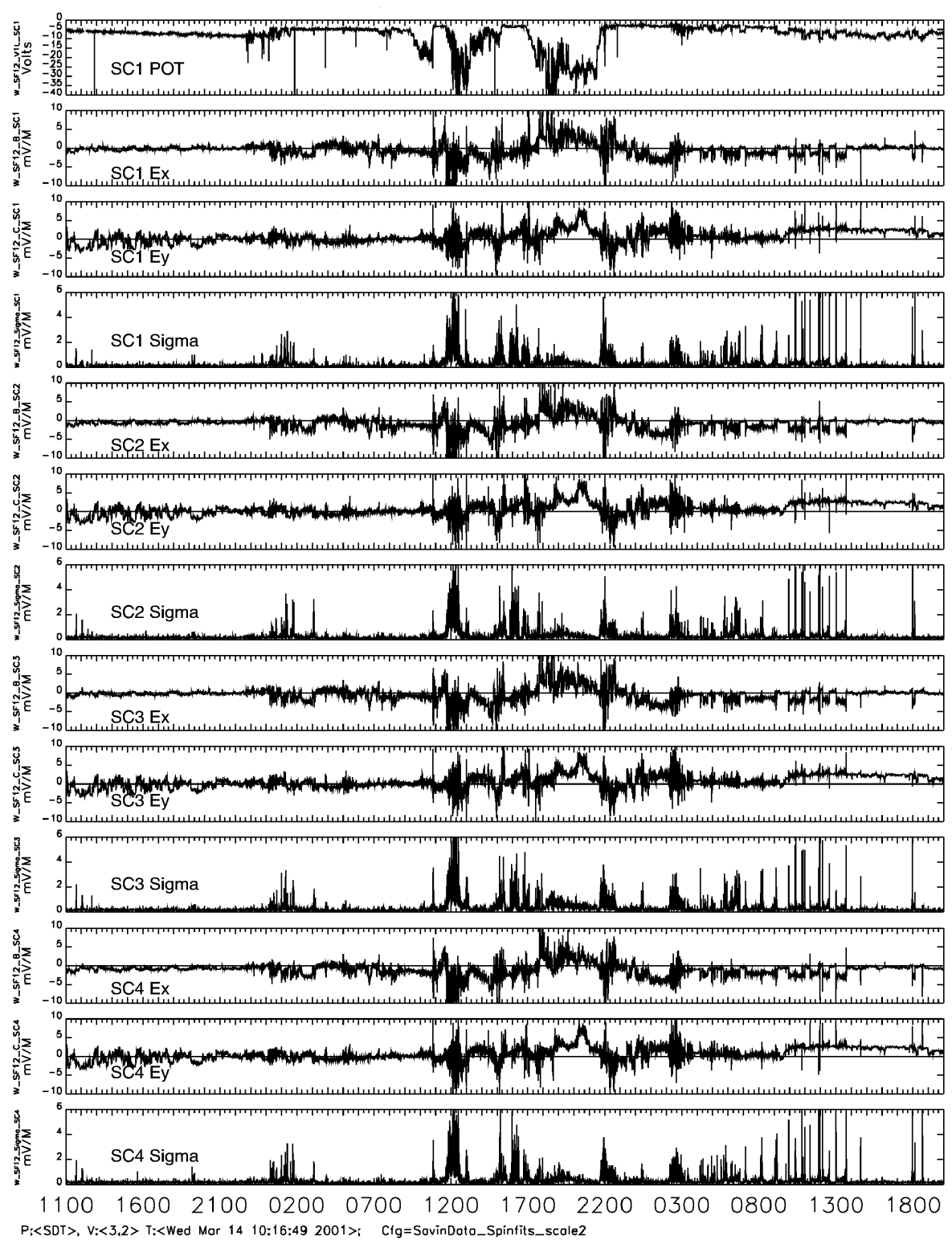

Fig. 1. A full orbit (57 hours) of EFW data from 22 January, 11:00 UT to 24 January, 19:00 UT. "Pot" is the probe-to-spacecraft voltage, while the other parameters are spin-period averages. $E_{X}$ and $E_{Y}$ are the electric field components in the directions of the projections of the GSE $X$ and $Y$ axes in the spin-plane, deviating a few degrees from the true GSE $X Y$ plane, while "Sigma" is the RMS deviation of the spin-fits, thus providing information about variations above the spin frequency. The figure shows raw data from onboard processing and a few glitches can be seen in the data.

produced by EFW.

The electron density and gradient of electron density are fundamental parameters where EFW can routinely produce spacecraft potential data with a time resolution of $0.2 \mathrm{~s}$. These data can be related to the electron density with good precision (Escoubet et al., 1997; Pedersen et al., 1995). A preliminary calibration curve for the Cluster instruments for this relation is discussed in Sect. 3 .

Electric field waveforms with frequencies below the spin frequency $(250 \mathrm{mHz})$, in many cases, show very close similarities between the four Cluster spacecraft in several of the plasma regions along the Cluster orbit. This opens the possibility for in depth studies of the structures. A few examples of structures with very high coherence ( 0.90 to 0.95 ) between the four spacecraft are discussed in Sect. 4.

Under magnetically quiet conditions, the Cluster satellites enter the plasmasphere with plasma that corotates with the Earth. The difference between the electric field from the four spacecraft is remarkably large near the border of the plasmasphere which shows that it is a very dynamic plasma region that deserves detailed studies. An example of a plasmapause crossing is discussed in Sect. 5. 
Crossings of auroral field lines by Cluster show high amplitude electric field variations. Fields of $100 \mathrm{mV} / \mathrm{m}$ have been seen that correspond to $1 \mathrm{~V} / \mathrm{m}$ in the ionosphere. A case of a convergent electric field was identified with a steep plasma density gradient. This case suggests an upward continuation of the quasi-static U-shaped potential which is associated with auroral arcs, as discussed in Sect. 6.

Data from the Cluster instruments offer tremendous possibilities for studying the magnetopause. In Sect. 7, a few examples of waves near the magnetopause are discussed primarily based on electric field data. Waves in the lower hybrid range of frequencies (approximately $10 \mathrm{~Hz}$ ) are suggested to be important for diffusion across the magnetopause and for onset of the reconnection. An event from the dawn side magnetopause is demonstrated. Kinetic Alfvén and drift waves are also identified in the magnetopause region and a case is discussed. It is argued that waves below the proton gyrofrequency could be identified as kinetic Alfvén waves with a wide range of spatial scales. The third example is a surface wave at the magnetopause with a wavelength of 6000 $\mathrm{km}$ moving with a phase velocity of $190 \mathrm{~km} / \mathrm{s}$ anti-sunward along the magnetopause.

A comparison was made between the plasma flow velocity measured in the upstream solar wind (at ACE spacecraft) during a northward turning of the magnetic field and the flow velocities estimated from the electric and magnetic field data from Cluster. The comparison of flows show that the electric field, its offset, and the spacecraft potential on Cluster are calibrated to an accuracy that is better than $10 \%$ for the example shown in Sect. 8.

Large electric field turbulence is observed at the cusp/magnetosheath interface. Both the orbit and the electric field data permit, for the first time, detailed studies of this turbulence. An example is discussed in Sect. 9.

\section{The EFW instrument}

\subsection{Introduction}

The Electric Field and Wave (EFW) instrument on Cluster consists of 4 spherical probes, $8 \mathrm{~cm}$ in diameter, at the end of long wire booms in the spin plane with a separation of $88 \mathrm{~m}$ between opposite probes. Each sphere can be operated in voltage mode (to obtain the average electric field between the probes in the spin plane) or current mode (to obtain the density). The voltage or current bias of the probes relative to the spacecraft is programmable and a current-voltage sweep can be made in both current and voltage modes for diagnostic purposes and to obtain the temperature and density of the ambient plasma. The analog signals pass through anti-aliasing filters before they enter the two 16-bit analog to digital converters operated at 36000 samples/s. The converters give a one-bit resolution for the low pass filters of $22 \mu \mathrm{V} / \mathrm{m}$, and $0.15 \mu \mathrm{V} / \mathrm{m}$ for the band pass filter. The digital output is finally routed via a 1 Megabyte internal memory to the telemetry. EFW has very flexible flight software with several programmable features, thus enabling a wide range of possibilities outside the standard operations described in this paper.

\subsection{Output data rate}

EFW has 4 output data rates, namely 1440, 15 040, 22240 and 29440 bits/s. These data rates are coordinated by the Master Science Plan and internally within the Wave Experiment Consortium (WEC). The telemetry packaging of the EFW data is made by the Digital Wave Processor (DWP) instrument (Wooliscroft et al., 1997).

\subsection{Modes of operation}

Each of the probes can operate in voltage or current mode. Together with the 4 output data rates and 5 filters (low pass filters at $10,180,4000,32000 \mathrm{~Hz}$, and a $50-8000 \mathrm{~Hz}$ band pass filter), this presents the opportunity for many combinations. The operation is primarily restricted by the telemetry available. There are, however, two main modes of operation. In normal mode telemetry (1440 bits/s), two electric field components in the spin plane are low-pass filtered at $10 \mathrm{~Hz}$ and sampled at 25 samples/s. With burst mode telemetry, the standard operational mode uses the same probe configuration but sampled at 450 samples/s and using the $180 \mathrm{~Hz}$ filter at a data rate of $15040 \mathrm{bits} / \mathrm{s}$. For both modes, the spacecraft potential at 5 samples/s and spin-fit electric field data (4 s resolution, see below) are available in housekeeping data. Spacecraft potential is important as it is a function of the electron density. The frequency range above $180 \mathrm{~Hz}$ can, however, only be recorded by the 1 Megabyte memory for short periods of time, but simultaneously with the normal and burst modes above.

In burst mode, the data rates of 22240 and $29440 \mathrm{bits} / \mathrm{s}$ may be used to transfer 3 or 4 signals sampled at 450 samples/s, respectively, at the expense of telemetry for STAFF and WHISPER. The signals from the probes through the 0$180 \mathrm{~Hz}$ filters can be measured either relative to the spacecraft or differentially between the two probes. The signals passing the $50-8000 \mathrm{~Hz}$ filters are only measured differentially with an additional gain of 148. All other signals are measured relative to the spacecraft (i.e. $10 \mathrm{~Hz}, 4 \mathrm{kHz}$ and $32 \mathrm{kHz}$ ). However, for the signal sampled at a rate of 25 samples/s (using $10 \mathrm{~Hz}$ filters), the potential difference between pairs of opposite probes is calculated on board before transmission to the ground. There is also a frequency counter with a $10-200 \mathrm{kHz}$ filter with a zero-crossing counter and an average-amplitude detector to monitor the power and frequency of the waves near the plasma frequency.

The flight software also incorporates a routine for deriving onboard spin-fits to the electric field data, thus providing two electric field components with a $4 \mathrm{~s}$ time resolution. The RMS deviation of the fit is also saved, giving a measure of wave activity above $0.25 \mathrm{~Hz}$. This amount of data is so small that it fits in the housekeeping data, and can thus be made available also outside the regular data acquisition periods. 


\subsection{Electric field measurements}

For the electric field measurements in the tenuous magnetospheric and solar wind plasmas, the probes should be given a constant bias current (Pedersen et al., 1998). The sphere potential is determined by the balance of plasma electron current, photoemission current, and a bias current to the sphere. The magnitude of the bias current controls the "working point" for the probe on the voltage-current characteristic curve and the aim is to find the working point that gives a low probe-to-plasma impedance.

Apart from providing the electric field, the probes in this mode are also very useful for providing data on the spacecraft potential. This information is normally available at 5 samples/s during data acquisition intervals, and at approximately 0.2 samples/s when only housekeeping data is available. As the spacecraft potential varies with the plasma density, this provides a density estimate in tenuous plasmas (Pedersen et al., 2001, this issue). In particular, it is very useful for identifying boundary transitions, and has been used extensively, for example on the Cluster quicklook parameter web page.

\subsection{Langmuir probe measurements}

The spherical sensors can be operated as current-collecting Langmuir probes to provide information on the plasma density and electron temperature. For this mode, the preamplifier is switched to a low impedance input and the probe is given a bias voltage rather than a bias current as for the electric field mode. The bias voltage is referred to as the satellite ground, though the variations in the spacecraft potential can be detected by another probe pair in electric field mode and errors from this source are consequently corrected. Ideally, a positively biased probe collects an electron current that is proportional to the plasma density, provided that the electron temperature is constant. Thus, variations in density will result in corresponding variations in the probe current. Also in this mode, it is important to control the photoelectrons in order to minimise the errors that they cause. This mode is, therefore, primarily useful in denser plasmas encountered by Cluster (e.g. solar wind, magnetosheath, plasmasphere). Probe bias sweeps in this mode are also very useful for monitoring the photoelectron saturation current of the probes, as well as for providing the plasma density and electron temperature (in the denser plasmas). Such sweeps are usually performed about once every two hours. It is also possible to do voltage mode (bias current) sweeps.

\subsection{New design of probe environment}

The sensor geometry is different from that flown on earlier missions and it produces a significantly improved measurement of the electric field. In previous instruments, each sphere was immediately adjacent to surfaces that were part of the boom wire and that were bootstrapped to certain potentials with respect to the sphere. Since these guards and stubs were adjacent to each sphere, there was an unavoidable flow of photoelectrons between the sphere and these surfaces. This produced a spurious sunward electric field signal since an asymmetry of $1 \%$ of the photoemission between the currents in opposite spheres results in an apparent electric field of several $\mathrm{mV} / \mathrm{m}$. To minimize this problem on Cluster, each sphere is held at the end of a thin wire at a distance of $1.5 \mathrm{~m}$ from the stubs and guards. This thin wire, which is electrically connected to its sphere, causes an improvement in the DC electric-field measurements by greatly diminishing stray currents to and from the sensors. It also improves the AC electric field measurement, particularly for the WHISPER and WBD instruments, since the capacitance between the sensor and the plasma is increased due to the thin wire. The success of the new design is witnessed by the unprecedently small spurious sunward electric field observed by EFW (of the order of afew tenths of $\mathrm{mV} / \mathrm{m}$ ) and the low noise levels and clean data of the WHISPER and WBD instruments (Décréau et al., 2001, this issue, Gurnett et al., 2001, this issue).

\section{The use of spacecraft potential for high time resolu- tion plasma density measurements}

Each electric field probe is biased with a current which forces electrons to the probe and brings the probe from its positive floating potential (no bias current) to a potential close to that of the ambient plasma. The probe can then serve as a reference for the spacecraft potential which is a function of emitted photoelectrons and the ambient electron density, and a weak function of the electron energy.

The spacecraft-to-probe potential difference, $V_{S}-V_{P}$, has previously been calibrated against plasma density on several spacecraft (Escoubet et al., 1997; Nakagawa et al., 2000; Scudder et al., 2000). Once calibrated, this technique is complementary to other plasma measurements since it is possible to measure density variations with a time resolution of the order of $0.1 \mathrm{~s}$, which is particularly important for Cluster boundary crossings. An additional advantage is the large density range covered, from approximately $10^{-3} \mathrm{~cm}^{-3}$ to about $10^{+2} \mathrm{~cm}^{-3}$.

A calibration of spacecraft potential measurements on Cluster has been done by data from the active plasma sounder Whisper (Fig. 2). So far, the calibration has only been carried out for $V_{S}-V_{P}$ up to +15 volts, corresponding to plasma densities of the order of $0.8 \mathrm{~cm}^{-3}$. Values of $V_{S}-V_{P}$ up to +60 volts have been measured over the polar caps and the lobes. For these values of $V_{S}-V_{P}$, the plasma density is of the order of $10^{-3} \mathrm{~cm}^{-3}$ and its determination will depend more on the electron energy than for smaller values of $V_{S}-V_{P}$ observed in the plasma sphere, magnetosheath and the solar wind. Later comparisons with calibrated particle experiments will make it possible to relate $V_{S}-V_{P}$ to plasma density for classes of electron energies in the plasmasphere and the lobes. The dotted lines in Fig. 2 shows an estimate of this relation based on earlier investigations. More details are given in Pedersen et al. (2001), this issue. Space- 
craft potential observations are used in several of the studies that follow.

\section{Examples of high coherence wave events along the Cluster orbit}

\subsection{Examples from spin-fit data}

The four spacecraft Cluster mission provides the first opportunity to determine the $3 \mathrm{D}$, time-dependent plasma characteristics. The present nominal inter-spacecraft separation is $600 \mathrm{~km}$ in regions with the tetrahedron configuration, which determines the scales for study with the four spacecraft. A major consideration for wave observations in a fast-flowing medium is the Doppler effect. Waveform data from the four spacecraft in a tetrahedral configuration allow for the correction of this effect when the wavelength is comparable with the inter-spacecraft separation. If the wavelength is small in comparison to this distance, then the determination of the wave normal directions on the four spacecraft yield information about the source location.

By looking at the electric field data from the four satellites over time periods of a few hours, it is easy to find structures that look similar from all four instruments not only near large-scale boundaries. Cross-correlation of spin averaged (4 s) electric field data over $3 \mathrm{~h}$ intervals may be as high as 0.95 between all four satellites. To obtain more detailed information on the coherency between spacecraft waveforms with periods longer than about $50 \mathrm{~s}$, electric field events are selected from regions of $6-16 R_{E}$ during the days between 19 and 23 January 2001. The orbit during this period had an apogee at about 14:30 LT.

There are four main frequency ranges available for electric field observations by EFW: the continuous spin-fit averaged data (4 s), normal mode data sampled at 25 samples/s, burst mode data sampled at 450 samples/s and internal burst mode data with sampling rates up to 36000 samples/s over short periods of time. Very often, high coherence between measurements in all these sampling ranges can be found between all four spacecraft.

Assuming that Cluster instruments observe plain waves of the form $\exp (k r-\omega t$,) one can see that the phase difference observed by each pair of satellites is $\Delta \varphi=k \Delta r$, where $\Delta r$ is the inter-spacecraft vector separation. From the observed phase shift, one can determine the $\boldsymbol{k}$ vector of the wave and the phase velocity. This information is then used to find the Doppler shift correction for the wave frequency $\Delta \varphi=k v$.

Events are selected in regions with large fluctuations in the electric field. The events are shown here to demonstrate the fact that on time scales of $50 \mathrm{~s}$ or longer, signatures with very high correlation between observations on the four spacecraft are found in many regions of the Cluster orbit. In Fig. 3, the $Y$-component (approximately in the GSE $Y$ direction) of the electric field for 8 events is shown. The $X$-component was not included here, but shows very much the same behaviour (see also Fig. 1 for an overview of 23 January).

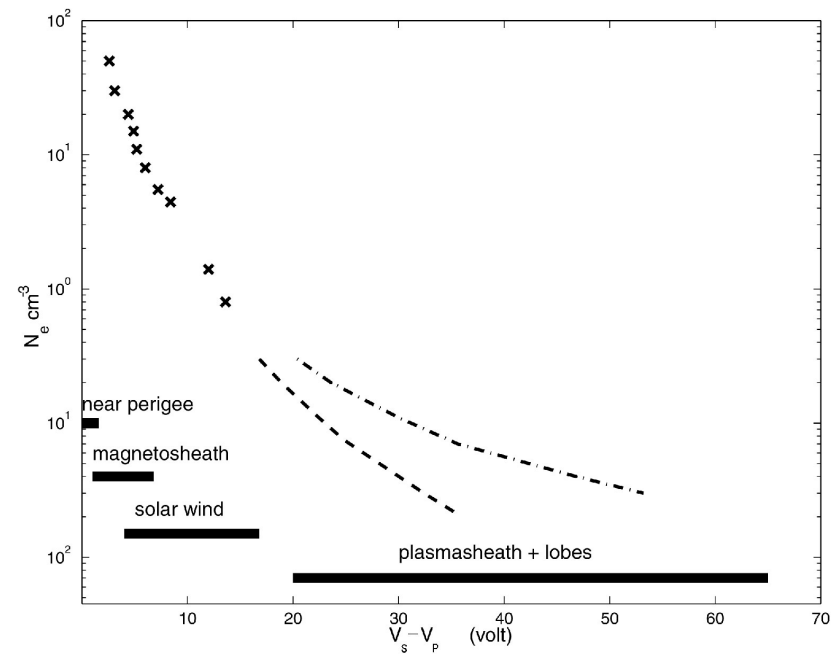

Fig. 2. Calibration of the spacecraft to-probe-potential difference $\left(V_{S}-V_{P}\right)$ in solar wind with electron densities measured by Whisper(crosses). Dotted lines indicate the expected range of $\left(V_{S}-V_{P}\right)$ in the plasmasheath and lobes, to be calibrated later with particle experiments.

Most of the events show periodic waveform structures with one or more dominant peaks in the frequency range from less than 1 to $15 \mathrm{mHz}$, as shown in Table 1 . During two of the events, 19 January, 07:10-07:44 UT and 23 January, 17:10-22:54 UT, there is a rather abrupt change from one frequency to a higher one, with the coherency equally high in both frequency ranges. Examples of two solitary structures are also included. A single pulse was observed on 23 January at 10:50 UT and a bipolar event was observed 23 January at 13:00. The pulse in the event on 23 January, 10:42-11:03 UT is a signature of compression. A solar wind shock was observed on ACE at approximately 10:00 UT with the parameters of density $N=10 \mathrm{~cm}^{-3}$, velocity $v=550 \mathrm{~km} / \mathrm{s}$ and magnetic field at $B=12 \mathrm{nT}$. Cluster was in the magnetosphere inside the magnetopause before compression and was brought into the magnetosheath.

These wave events were chosen to illustrate that high coherence electric field events between Cluster spacecraft occur in many regions along the orbit and for many different waveforms. Further studies will be made to obtain the detailed characteristics and sources of these wave phenomena, by including data from other instruments on Cluster and data from possible ground-based effects.

\subsection{Example of analysis of internal burst mode data}

The internal burst mode capability of EFW' which allows for the synchronized sampling of both spin plane $E$-field components, opens a window in physics in the VLF regime which is comparable with the STAFF SA instrument but the EFW burst data show waveform structures which allow for a high degree of flexibility during post-processing. One way of visualizing burst mode data is in terms of the polarization of the spin plane electric field. In Fig. 4, we see an example of the 

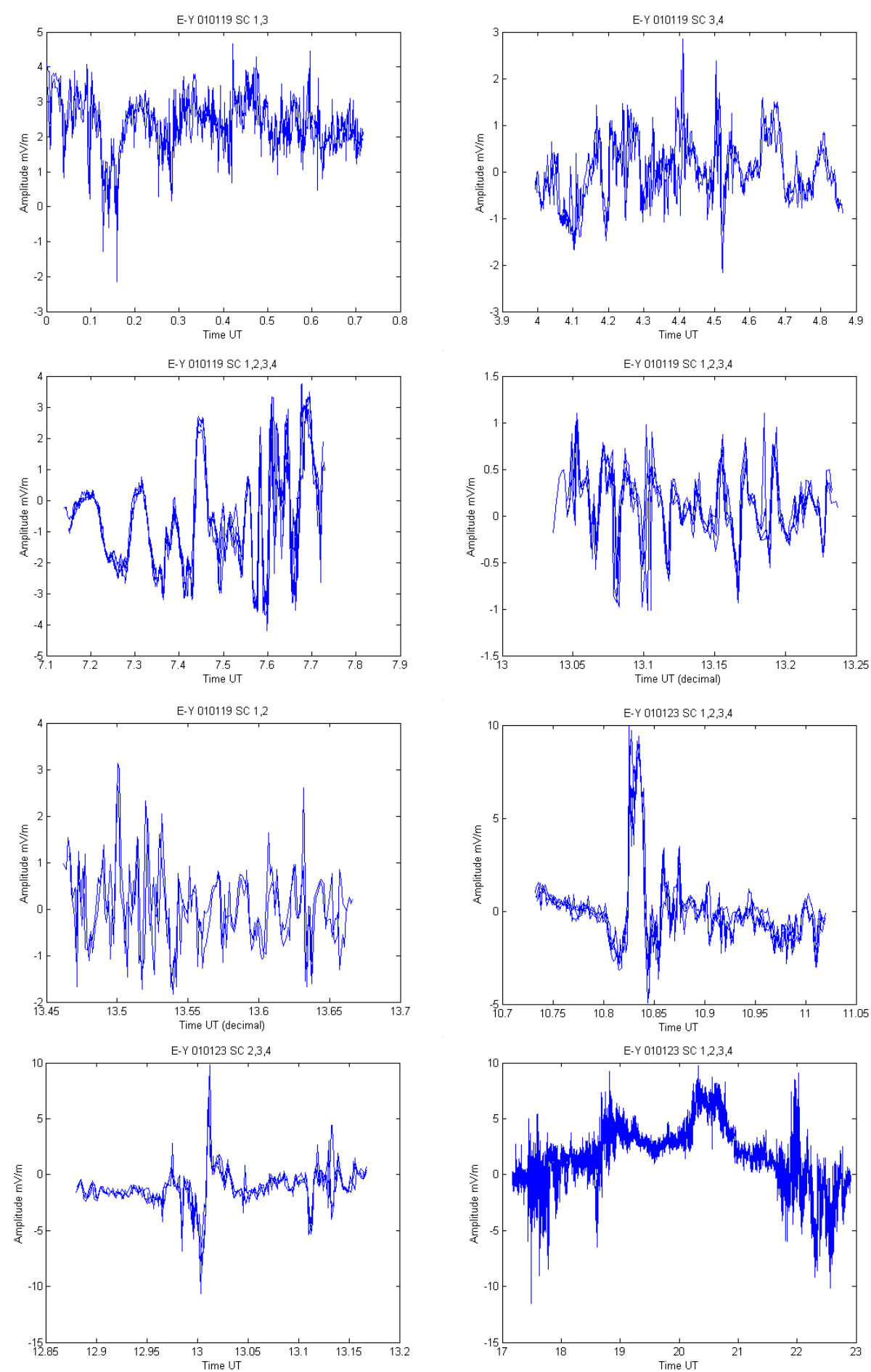

Fig. 3. Time series plots from 8 events based on spin-averaged $Y$-component electric field data. The overlay data from up to four spacecraft show good coherence over distances of several hundred $\mathrm{km}$. Note that the time is given in hours and decimal of hours.

polarization concurrently from two of the Cluster spacecraft. The data was recorded when Cluster was in the solar wind and shows some distinct localized patches of electrical activity. There is no obvious correlation in the activity between the spacecraft measurements, indicating a spatial extent of less than the spacecraft separation.
The polarization parameters are defined according to

$$
\begin{aligned}
& I=\left\langle\left(\left|E_{X}\right|^{2}+\left|E_{Y}\right|^{2}\right) / 2\right\rangle \\
& v=(p V) /(2 I) \\
& \phi=\arctan (U / Q) / 2 \\
& p=\sqrt{\left.Q^{2}+U^{2}+V^{2}\right)} /(2 I)
\end{aligned}
$$


Table 1. Wave event characteristics

\begin{tabular}{|c|c|c|c|c|}
\hline Event & Location SC 1 & Region & Spectrum & Phase \\
\hline $\begin{array}{l}\text { 19 Jan } \\
00: 00-00: 43 \text { UT }\end{array}$ & $\begin{array}{l}00: 24 \mathrm{UT}, 6.10 R_{E} \\
{[-2.59,0.66,5.48] \mathrm{GSE}}\end{array}$ & $\begin{array}{l}\text { Auroral oval or } \\
\text { Plasmasphere }\end{array}$ & $\begin{array}{l}\text { Single peak at } \\
2 \mathrm{mHz}\end{array}$ & $\begin{array}{l}\text { Approx. const. } \\
\text { but irregular }\end{array}$ \\
\hline $\begin{array}{l}19 \text { Jan } \\
04: 01-04: 52 \text { UT }\end{array}$ & $\begin{array}{l}04: 24 \mathrm{UT}, 10.46 R_{E} \\
{[1.17,5.63,8.74] \mathrm{GSE}}\end{array}$ & $\begin{array}{l}\text { Plasmasheath/lobe } \\
\text { boundary }\end{array}$ & $\begin{array}{l}4 \text { Peaks at } 1 \text {, } \\
2,5,8 \mathrm{mHz}\end{array}$ & $\begin{array}{l}0.05 \mathrm{~Hz} \text { for } \\
0-180^{\circ}\end{array}$ \\
\hline $\begin{array}{l}\text { 19 Jan } \\
07: 10-07: 44 \text { UT }\end{array}$ & $\begin{array}{l}07: 30 \mathrm{UT}, 13.14 R_{E} \\
{[4.06,8.37,9.28] \mathrm{GSE}}\end{array}$ & $\begin{array}{l}\text { Magnetosphere } \\
\text { lobe/plasmasheath }\end{array}$ & $\begin{array}{l}2 \text { Peaks at } 10 \text {, } \\
1.5 \mathrm{mHz}\end{array}$ & $\begin{array}{l}0.04 \mathrm{~Hz} \text { for } \\
0-180^{\circ}\end{array}$ \\
\hline $\begin{array}{l}\text { 19 Jan } \\
\text { 13:03-13:14 UT }\end{array}$ & $\begin{array}{l}\text { 13:08 UT, } 16.65 R_{E} \\
{[8.47,11.61,8.41] \mathrm{GSE}}\end{array}$ & $\begin{array}{l}\text { Solar wind/ } \\
\text { magnetosheath }\end{array}$ & $\begin{array}{l}\text { Peak at } \\
15 \mathrm{mHz}\end{array}$ & $\begin{array}{l}\text { Approx. const. } \\
\text { but irregular }\end{array}$ \\
\hline $\begin{array}{l}19 \text { Jan } \\
13: 28-13: 45 \text { UT }\end{array}$ & $\begin{array}{l}13: 34 \mathrm{UT}, 16.85 R_{E} \\
{[8.76,11.78,8.28] \mathrm{GSE}}\end{array}$ & Solar wind & Peak at $2 \mathrm{mHz}$ & $\begin{array}{l}0.09 \mathrm{~Hz} \text { for } \\
0-1806^{\circ}\end{array}$ \\
\hline $\begin{array}{l}23 \text { Jan } \\
10: 42-11: 03 \text { UT }\end{array}$ & $\begin{array}{l}\text { 10:50 UT, 8.71 } R_{E} \\
{[5.18,0.32,-6.99] \mathrm{GSE}}\end{array}$ & $\begin{array}{l}\text { Magnetosphere/ } \\
\text { magnetosheath }\end{array}$ & $\begin{array}{l}\text { A single } \\
\text { structure }\end{array}$ & $\begin{array}{l}\text { Const phase } \\
\text { to } 0.06 \mathrm{~Hz}\end{array}$ \\
\hline $\begin{array}{l}23 \text { Jan } \\
12: 51-13: 12 \mathrm{UT}\end{array}$ & $\begin{array}{l}13: 00 \mathrm{UT}, 6.01 R_{E} \\
{[1.74,-1.61,-5.78] \mathrm{GSE}}\end{array}$ & $\begin{array}{l}\text { Near Southern } \\
\text { auroral oval }\end{array}$ & $\begin{array}{l}\text { Bidirectional } \\
\text { structure }\end{array}$ & \\
\hline $\begin{array}{l}23 \mathrm{Jan} \\
\text { 17:10-22:54 UT }\end{array}$ & $\begin{array}{l}20: 00 \mathrm{UT}, 7.74 R_{E} \\
{[-1.15,2.73,7.15] \mathrm{GSE}}\end{array}$ & $\begin{array}{l}\text { North auroral oval } \\
\text { to magnetosheath }\end{array}$ & $\begin{array}{l}90 \mathrm{~min} \text { period } \\
\text { and } 0.8 \mathrm{mHz}\end{array}$ & $\begin{array}{l}0.09 \mathrm{~Hz} \text { for } \\
0-180^{\circ}\end{array}$ \\
\hline
\end{tabular}

$Q=\left\langle\left|E_{X}\right|^{2}-\left|E_{Y}\right|^{2}\right\rangle$

$U=2\left\langle R_{E}\left(E_{X} E_{Y}^{*}\right)\right\rangle$

$V=2\left\langle\operatorname{Im}\left(E_{X} E_{Y}^{*}\right)\right\rangle$

and $E_{X}, E_{Y}$ are electric field components in GSM coordinates and the brackets \langle\rangle denote time averaging (we assume a time dependence of $\propto \exp (+i \omega t)$. The four parameters of the electric field in the spin plane are $I$ the power, $v$ the degree of ellipticity, $\phi$ the tilt angle, and $p$ the degree of polarization. The sign of $v$ is such that positive values correspond to a positive sense of rotation of the electric field along the $Z$-axis. The tilt angle is relative to the positive $X$-axis. They are related to the Stokes parameters but do not necessarily refer to the transverse electric field components of an electromagnetic wave.

A remarkable feature of the polarization spectra of this electrical activity is that for any given time and spacecraft, the polarization is virtually identical over the entire frequency band of the EFW burst mode. By comparison, at times when low electric power is observed, no particular polarization is favored as expected, indicating good instrumental conditions for such polarization measurements. Furthermore, the ellipticity shows that the electric field is consistently linearly polarized to within a few percent with no exceptions.

\section{EFW observations in the plasmasphere}

Near the perigee passes, the Cluster spacecraft have quick encounters with the inner magnetosphere down to $L=4$, during which the satellites often skim the plasmapause region.
This boundary usually appears as a steep density decline, typically at about $L=3-6$, but for disturbed conditions it may move within $L=3$, whereas for very quiet conditions it can expand to $L=12$ (Laakso and Jarva, 2001).

Under magnetically quiet conditions (approximately $K p<3$ ), the Cluster satellites usually cross the plasmapause and enter the plasmasphere. This region, consisting of thermal plasma escaping from the ionosphere, appears as a dense torus around the Earth, where the charged particles rotate around the Earth at the same angular velocity as the Earth. This corotation of the plasmasphere is accompanied by an earthward pointing corotation electric field that forms closed equipotentials encircling the Earth (Volland, 1984). In fact, in a quasi-static model, the plasmapause can be defined as the last closed equipotential in a steady-state magnetosphere with a constant convection field (Lemaire and Gringauz, 1998).

For increasing magnetic activity, magnetospheric convection is enhanced and the location of the flow separatrix moves rapidly earthward. In such conditions, the outer plasmasphere becomes strongly structured, and plasmaspheric material will appear beyond the plasmapause. Such dense plasma elements become detached from the plasmasphere and drift away under the influence of the magnetospheric convection (Carpenter and Lemaire, 1997).

The inner magnetosphere is not considered a key region for the Cluster mission, and as a result there are not many intervals yet where simultaneous plasmaspheric observations are collected by all four spacecraft. Figure 5 shows an example of such measurements with the EFW experiment, on 28 January 2001, 09:25-10:30 UT. Unfortunately, the data collection was turned off just before the second plasmapause 
Cluster S/C 1, polarization of despun spin-plane E-field

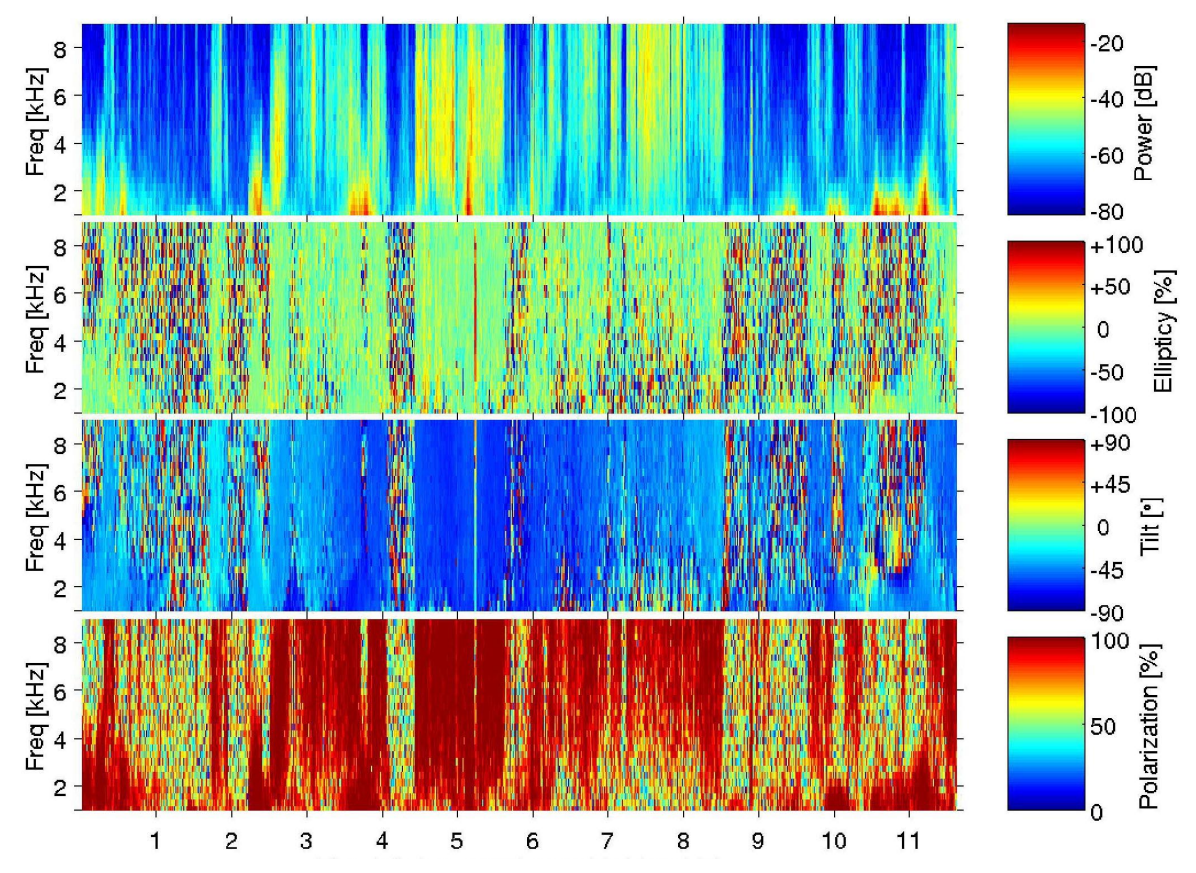

Cluster S/C 2, polarization of despun spin-plane E-field

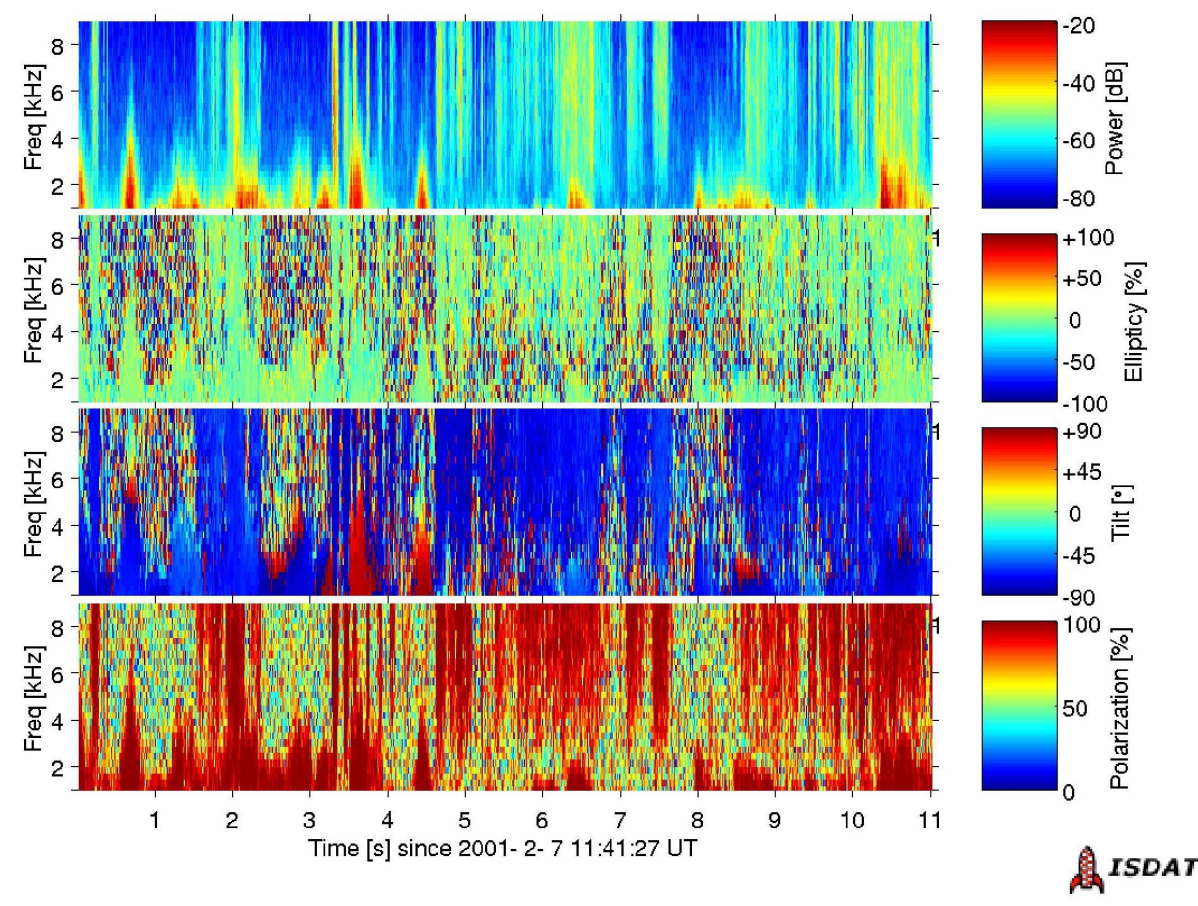

Fig. 4. Polarization plots of internal memory burst data. The panels of polarization are from top to bottom, respectively, the total power $I$, the degree of ellipticity $v$, the tilt angle $\phi$, and the degree of polarization $p$.

crossing, soon after 10:30 UT. The panels from top to bottom are the electric field $E_{X}$ and $E_{Y}$ components in the magnetic field line coordinate system and the potential difference between a biased probe and the spacecraft; the field line coordinates are defined so that the $Z$ coordinate is along the magnetic field $B$, the $X$ coordinate is in the plane of the magnetic field line and perpendicular to $Z$, pointing generally towards the equator, and the $Y$ coordinate completes the frame of reference and points generally westward.

In this example, the four satellites cross the plasmapause within six minutes. The measurements were taken in the post-midnight sector at 03:00-04:00 MLT, where the plasma- 
pause is known to experience large changes in its geocentric distance (Laakso and Jarva, 2001). The density profiles may look quite similar, but a closer examination of the observations reveals plenty of discrepancies in the region just outside the plasmapause. Such irregularities are quite common for disturbed intervals, and this example was taken during moderate geomagnetic disturbances, as indicated by the preliminary AE index (data not shown here).

The $E_{X}$ component is responsible for the corotation flow, and at this altitude, the corotation electric field $\left(E_{c o r}\right)$ is expected to be $0.9-1.0 \mathrm{mV} / \mathrm{m}$ and its value varies with distance $(r)$ as $r^{2}$; the corresponding drift velocity is about $2 \mathrm{~km} / \mathrm{s}$ eastward. The top panel shows that outside the plasmapause, the convection electric field $E^{c o n}$ is significantly larger than $E_{c o r}$, and the convection direction is primarily eastward, as expected in this local time sector. The $E_{Y}$ component, displayed in the middle panel, yields roughly the radial drift velocity so that a negative value indicates an outward drift velocity. It vanishes in the plasmasphere, but from the outer plasmaspheric regions into the convecting magnetosphere, it becomes increasingly important. The differences between the electric field measurements on the four satellites are quite remarkable, which only tells us that the outer plasmasphere is dynamically a very interesting plasma region, which deserves more multi-point measurement intervals with the Cluster satellites.

\section{Large-amplitude auroral electric fields observed by Cluster near perigee}

Large-amplitude electric fields represent a common feature on auroral field lines at altitudes above a few thousand kilometers, as shown by a number of spacecraft such as Dynamics Explorer 1, Viking, Polar and FAST and in the return current region at altitudes as low as $800 \mathrm{~km}$, as was first shown by Freja (Marklund et al., 1997). In many studies, such observations have been interpreted in terms of quasi-static fieldaligned electric fields (directed upward and downward in the upward and downward current regions, respectively) located below or partly below the spacecraft. In a recent study, largeamplitude electric fields observed by Polar were interpreted as signatures of Alfvén waves carrying enough Poynting flux to produce intense auroral arcs (Wygant et al., 2000).

The objective of the present study is to investigate the electric field on and adjacent to auroral field lines using data obtained near perigee, where the four Cluster spacecraft are aligned roughly as pearls on a string. With a time separation of a few minutes between the Cluster spacecraft passing across the auroral field lines, the spatial and temporal characteristics of the electric fields can be investigated in much greater detail than from the single satellite observations reported in the past. The long-term objective of this study is to gain insight about the energy transport between the magnetosphere and the auroral ionosphere, whether it may be described by a quasi-static current circuit or by an Alfvén wave scenario. This is a fundamental question which, however,

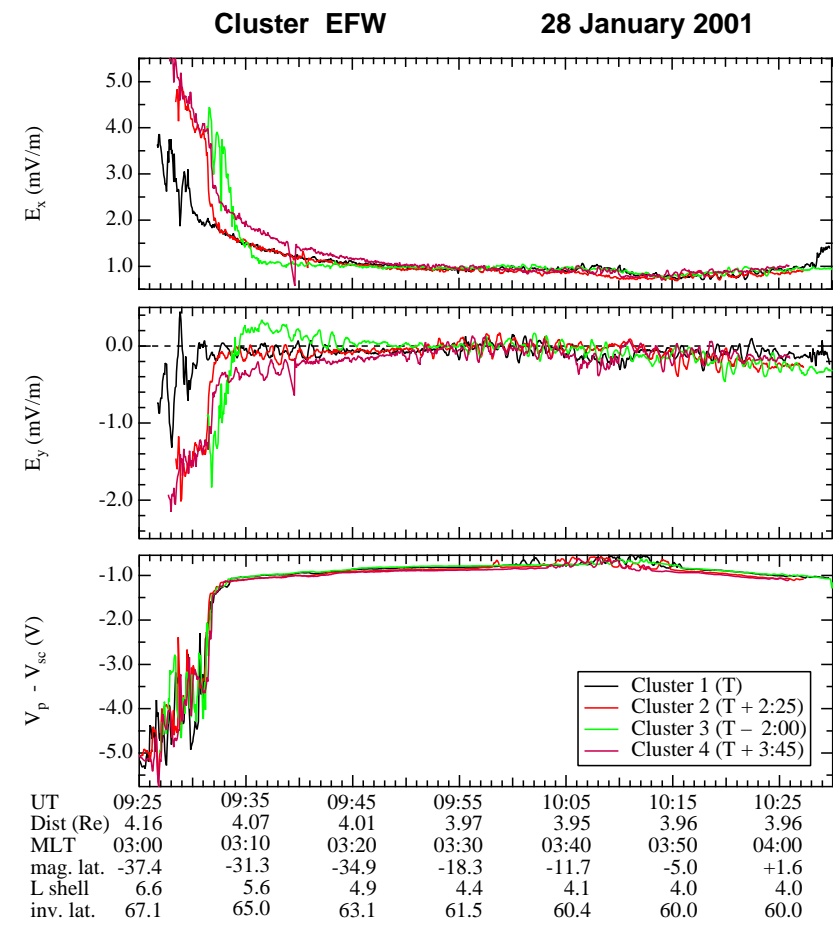

Fig. 5. Observations by the EFW experiment in the inner magnetosphere on 28 January 2001, 09:25-10:30 UT. The two electric field components in the field line coordinate system and the differential potential measurements are shown. The plasmapause is crossed by Cluster1 at $\sim 09: 32$ UT and by the other three spacecraft within \pm 4 min of that time, as shown by the legend in the bottom panel.

requires the detailed analysis of magnetic field and particle data, in addition to the electric field data. This analysis is outside the scope of this initial study.

The data coverage for auroral field line crossings near perigee has so far been quite limited. To date, there are only some ten events available with good data coverage from auroral crossings of which, we will focus here on one event that took place on 14 January 2001, around 04:30 UT during moderate substorm activity. The AL and AU indices peaked at values around $300 \mathrm{nT}$ at 04:00 UT and the Polar UVI images showed at the same time extended nightside auroral activity (data not shown here). The activity then decreased such that at the time of the oval crossings by the Cluster satellites around 04:30 UT, the AL and AE indices had decreased to about half of these values and the auroral oval was more diffuse. Figure 6 shows the relative positions of the four spacecraft in a plane perpendicular to $B$, with the vertical and horizontal axes showing the distances in the poleward and eastward directions, respectively. Note that satellites 1 , 3 , and 4 are almost aligned along the direction of the velocity component perpendicular to $B$ (indicated by the dotted line). The times of the consecutive crossings relative to spacecraft 3 are $105 \mathrm{~s},-90 \mathrm{~s}$, and $-182 \mathrm{~s}$ for spacecraft 1 , 2 , and 4 , respectively. Thus, the time separation is roughly 90-100 s between each of the satellites which, if mapped to the ionosphere, corresponds to distances perpendicular to $B$ 

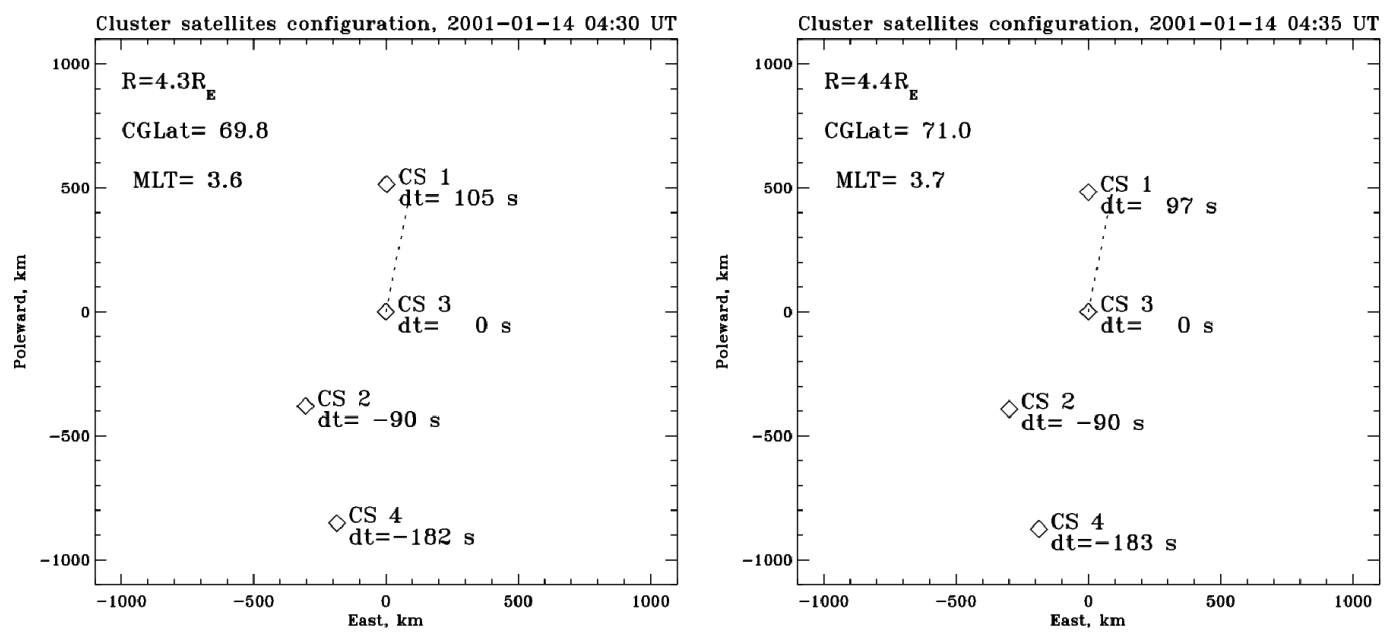

Fig. 6. Relative positions of the four Cluster spacecraft in a plane perpendicular to $B$ at 04:30 and 04:35 UT on 14 January 2001. Vertical and horizontal axes represent distances in the geomagnetic poleward and eastward directions. Spacecraft 3 is located at the center. The time differences $(d t)$ between the four spacecraft to that of spacecraft 3 are indicated at the spacecraft positions. The geocentric distance, corrected geomagnetic latitude and magnetic local time for spacecraft 3 are given at the top, left. The dotted line indicates the direction of the velocity component perpendicular to $B$ for spacecraft 3 .

of roughly 40-50 km. In the upper four panels of Fig. 7, one can see the sunward component of the electric field in the spin plane versus time after 04:27 UT for each of the four spacecraft, followed by four plots of the duskward electric field component and four plots of the negative of the satellite potential as derived from the EFW data (for a description of the EFW instrument, see above).

To be able to directly compare the data between the four spacecraft, the time lines for satellite 1,2 , and 4 have been shifted relative to the reference time line for satellite 3 by the numbers given in Fig. 6. Note that the entry and the exit out from the region which is characterized by the presence of small-scale irregular electric field variations and a decrease in the satellite potential (decrease in plasma density), is roughly the same for spacecraft 2,3 , and 4 and somewhat displaced for spacecraft 1 . This might be explained by an equatorward motion of the auroral oval during the time span between the oval entries by spacecraft 1 and spacecraft 3 . The time between the entry and the exit of the region which is characterized by irregular electric fields and decreases in the satellite potential is roughly the same for all spacecraft, around $700 \mathrm{~s}$, which corresponds to a $350 \mathrm{~km}$ distance in the ionosphere or a latitudinal width of $3.2^{\circ}$. Polar UVI imager data from this time and the local time sector (not shown here) around 03:30 MLT indicate an oval width of 5-6 and that the equatorward edge of the oval is located at $69.5^{\circ}$. This is in good agreement with the location of the Cluster spacecraft at the entry into the region of irregular fields. Thus, the irregular electric fields seem to dominate within the central and equatorward portions of the oval, i.e. primarily within the large-scale region 2 upward field-aligned currents. Note that the sunward electric field component is much weaker on spacecraft 4 than on spacecraft $1-3$. The same is true for the satellite potential which is much weaker (larger electron density) and flatter on spacecraft 4 than on spacecraft 1-3.

What persistent or repetitive features observed by two or more spacecraft can we distinguish from these electric fields and satellite potential traces? Moving from left to right, the entry into the oval is characterized by the onset of electric field fluctuations and a slow decrease in the negative of the satellite potential. Next, a region of very bursty electric fields reaching magnitudes exceeding $100 \mathrm{mV} / \mathrm{m}$ on spacecraft 4 (duskward component) are observed by spacecraft 3 almost simultaneously at around $T=260 \mathrm{~s}$ (marked with a vertical line A in Fig. 7) and about $10 \mathrm{~s}$ and $25 \mathrm{~s}$ later on spacecraft 2 and spacecraft 1, respectively. The most pronounced and persistent feature is the intense converging electric field structure seen by spacecraft 1,2 , and 3 at around $T=375 \mathrm{~s}$ (marked with line B in the figure). It is colocated with a steep gradient in the satellite potential, and thus, a gradient in the plasma density (most pronounced on spacecraft 3 ). The fact that the field is convergent indicates that the structure is related to an upward field-aligned current, presumably associated with an arc. The peak electric field reaches $90 \mathrm{mV} / \mathrm{m}$ on spacecraft 2 and 3 and $55 \mathrm{mV} / \mathrm{m}$ on spacecraft 1 . The width of the structure ranges between 10 and $15 \mathrm{~km}$ mapped to the ionosphere, which is consistent with the characteristic scale size of discrete auroral arcs. Finally, between 730 and $780 \mathrm{~s}$ (marked with the vertical lines $\mathrm{C}$ and $\mathrm{D}$, respectively, in Fig. 7) on spacecraft 2, 3 and 4 and about 25 s later on spacecraft 1, a small positive duskward electric field is observed, i.e. the electric field has the same direction as that which is typical of the polar cap. This duskward electric field interval was preceded by a region of irregular electric fields.

In summary, the characteristics of the auroral electric field at an altitude of about $3.3 R_{E}$ have been examined based on data from the Cluster EFW instrument from an auroral field line crossing near-perigee when the four spacecraft were 


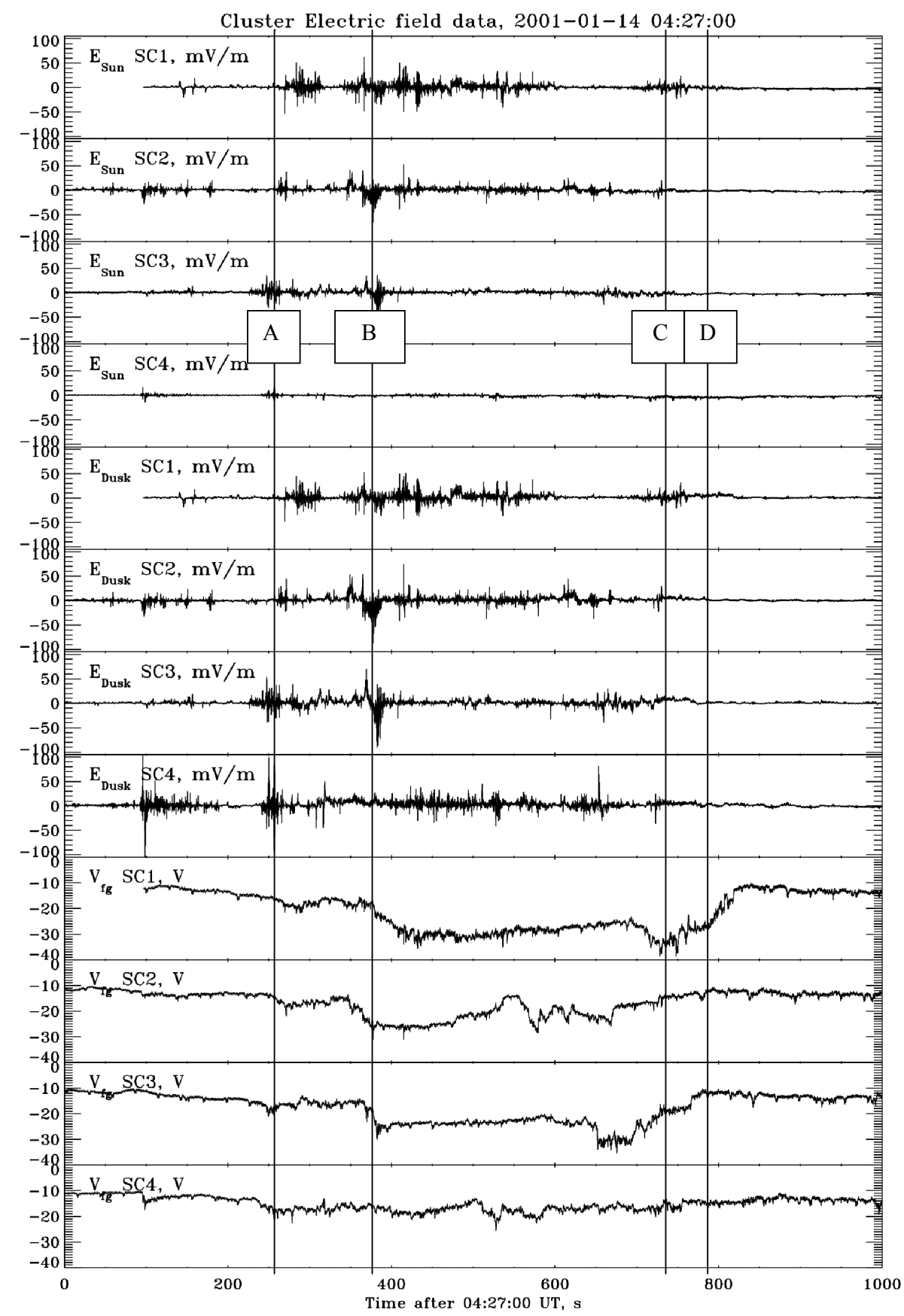

Fig. 7. Electric field and satellite potential data from near-perigee auroral field line crossings by the four Cluster spacecraft on 14 January 2001. The time axis is seconds relative to 04:27 UT on spacecraft 3, while the time axes for the other spacecraft have been shifted. The vertical lines A-D at 260, 375, 730 and $780 \mathrm{~s}$, respectively, are included to facilitate the location of regions described in the text.

aligned roughly as pearls on a string. The geophysical data available indicate that the crossing took place during the recovery phase of an auroral substorm peaking in activity about half an hour prior to the crossing. Although there are differences seen particularly in the electric field fine structure, common large-scale features are seen both in the electric field and in the satellite potential that persist during the time span of $287 \mathrm{~s}$ between the crossings of spacecraft 1 and spacecraft 4. The entry and the exit of the region characterized by irreg- ular and relatively intense electric fields and negative values of the satellite potential (indicative of low plasma densities) occurred at the same geographic position on spacecraft 2-4 and somewhat more poleward on spacecraft 1 , indicating a fairly stable oval, as verified also by the Polar UVI images. The region of irregular electric fields was located within the central and equatorward portion of the morning-side auroral oval, i.e. within the region 2 upward current region. Other persistent features seen by three or four spacecraft were a re- 


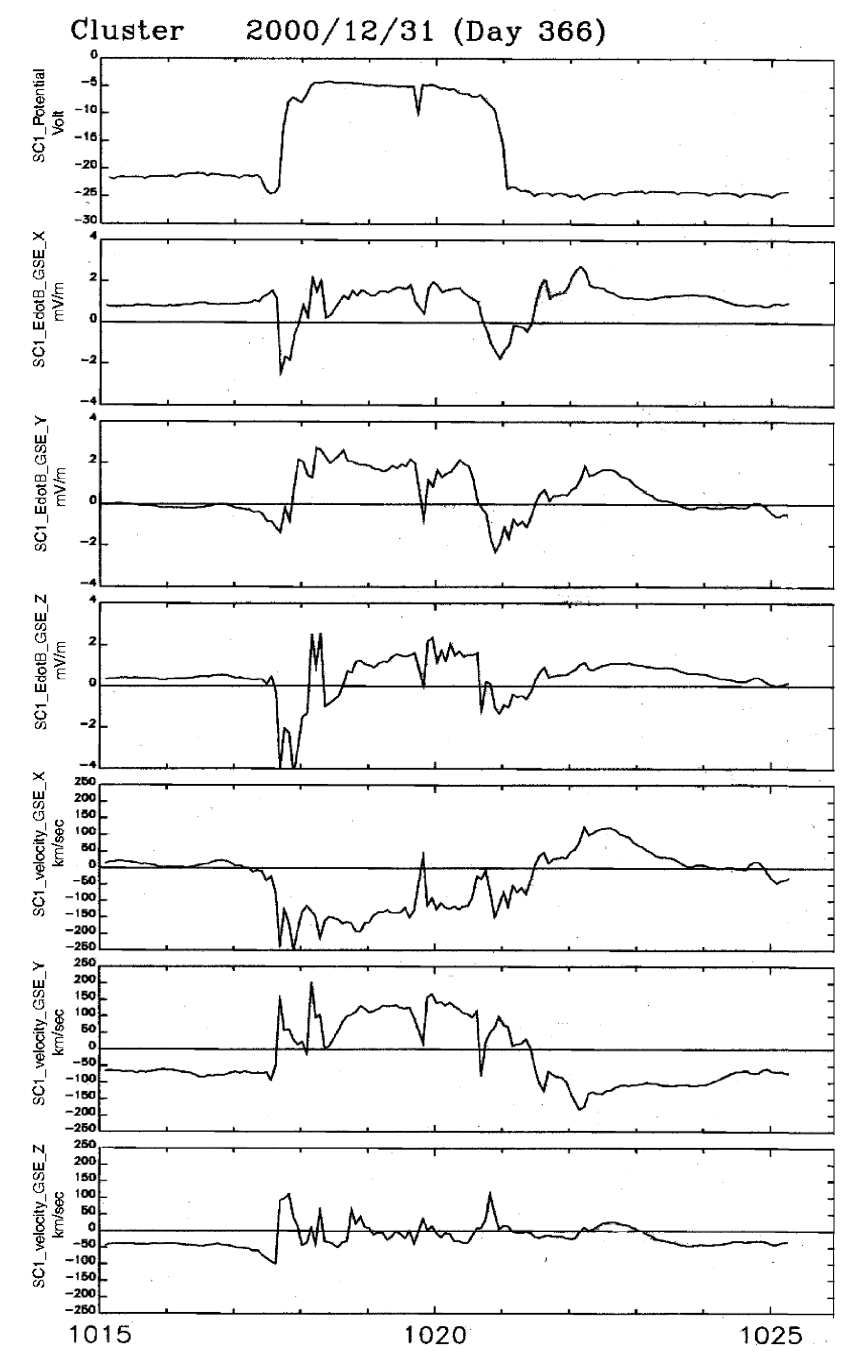

Fig. 8. An example of a magnetopause crossing (between 10:1510:25 UT) where the spacecraft entered the magnetosheath and returned three minutes later. The figure demonstrates the detailed behaviour of the spacecraft potential (plasma density), electric field and plasma flow near the boundary.

gion of bursty electric fields reaching above $100 \mathrm{mV} / \mathrm{m}$ on spacecraft 4 located about $50 \mathrm{~km}$ equatorward of the convergent electric field structure. Moreover, a region of irregular electric fields followed by an increase in the duskward electric field component was observed near the poleward edge of the oval where the electric field was changing to that which is characteristic of the polar cap.

The most pronounced feature was a convergent electric field structure seen by spacecraft $1-3$, but most pronounced on spacecraft 2 and 3 with peak values of $90 \mathrm{mV} / \mathrm{m}$ which, if mapped to the ionosphere, would amount to almost $1 \mathrm{~V} / \mathrm{m}$. The structure, with a width of $10-15 \mathrm{~km}$ mapped to the ionosphere, was colocated with a steep gradient in the plasma density and presumably associated with the upward fieldaligned current of a discrete arc within region 2 . The fact that this structure retained its major features for more than three minutes strongly suggests that it represents the upward continuation of a quasi-static U-shaped potential contour associated with an arc. The persistency of this $10-15 \mathrm{~km}$ electric field structure thus provides support for a quasi-static auroral potential structure that extends at least to a geocentric distance of $4.3 R_{E}$.

\section{Magnetopause crossings}

\subsection{General}

Cluster experienced numerous magnetopause crossings 31 December 2000 between 10:00 and 14:30 UT on the dusk flank ( 17:00 LT). Geotail was close to the bow shock in the solar wind at approximately 07:00 LT. The solar wind speed was $320 \mathrm{~km} / \mathrm{s}$ and the plasma density $5 \mathrm{~cm}^{-3}$. The magnetic field $B_{Z}$ component varied from north to south with a magnitude of 3-4 nT.

Some of the passages of Cluster into the magnetosheath showed a positive $B_{Z}$ and others have a negative value. The latter case is chosen as an example in Fig. 8. The upper panel demonstrates that the density increased to magnetsheath values at 10:17:40, about $10 \mathrm{~s}$ before $B_{Z}$ started to turn southward. The most interesting part is that $E_{Y}$ is positive in the current layer which can be estimated to be in the $-X$ and $Y$ direction. The current layer, as seen in the magnetic measurements (not shown here), was at about 10:17:50 to 10:18:10 for the magnetopause inward motion, and at 10:20:35 to 10:20:55 for the outward motion. This indicates that $\boldsymbol{E} \bullet I$ is positive and that electromagnetic energy is transformed to kinetic energy, a signature of reconnection.

The outward motion of the magnetopause does not have the same electric field signature and it is difficult to estimate $\boldsymbol{E} \bullet I$. The density drops in the current layer and there appears to be no dense plasma inside the current layer in this case.

This example is meant to illustrate the tremendous possibilities of studying the magnetopause with complete analysis of magnetic and electric fields, and particles on all four spacecraft.

7.2 Multipoint observations of waves in the lower hybrid frequency range near the magnetopause

Waves in the lower hybrid range of frequencies are often observed near the magnetopause. These waves may be important for diffusion across the magnetopause, and for the onset of reconnection (see e.g. Sibeck 1999). Figure 9 shows the data from the four Cluster satellites near the duskward magnetopause. The upper panel shows an estimate of the negative of the spacecraft potential, which depends on the density in such a way that a maximum (minimum) on the curve corresponds to a maximum (minimum) of the density. The satellites enter from the low density magnetosphere into a denser boundary layer at two instances before 15:03:30 UT. The satellites then enter the magnetosheath (or rather the motion of the magnetopause brings the satellites into the magnetosheath). Three satellites then stay in the magnetosheath until around 15:05:40 UT, and then stay in a boundary layer 


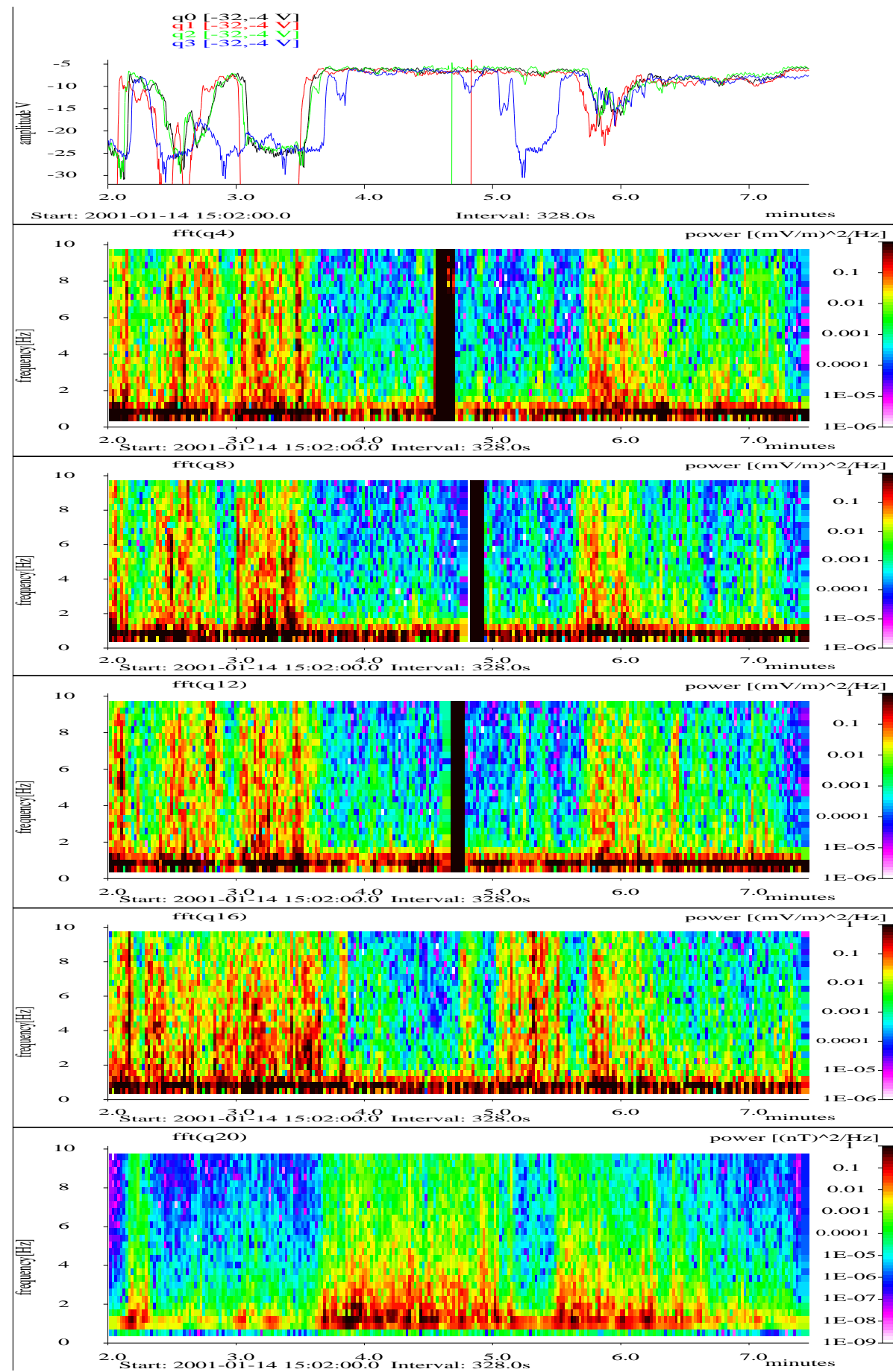

Fig. 9. Data from the four Cluster spacecraft near the dusk magnetopause. The top panel is the negative of the spacecraft potential, panels 2-5 are the electric field data from spacecraft $1-4$ and the bottom panel is the magnetic field data obtained by STAFF.

for some time. Satellite 4 leaves the magnetosheath for about half a minute after 15:05:00. Panels 2-5 show EFW electric field data from satellites 1 to 4 at frequencies up to $10 \mathrm{~Hz}$. It is clear that broadband (in the satellite frame) waves are seen on all spacecraft at the density gradients and in the lower density magnetosphere and boundary layer regions. (The signal up to around $1 \mathrm{~Hz}$ in this figure is partly due to the quasi-static electric field) In particular, note the waves after 15:05:00 UT in panel 5, when satellite 4 leaves the magnetosheath for about half a minute. The bottom panel shows magnetic field oscillations up to $10 \mathrm{~Hz}$ obtained by the STAFF instrument on satellite 4 . Comparing the two lowest panels of Fig. 9, we find that the strong electric and magnetic oscillations at a few $\mathrm{Hz}$ clearly are anti-correlated, the former is confined to the magnetosphere and the Earthward boundary of the magnetopause, and the latter is occurring primarily 


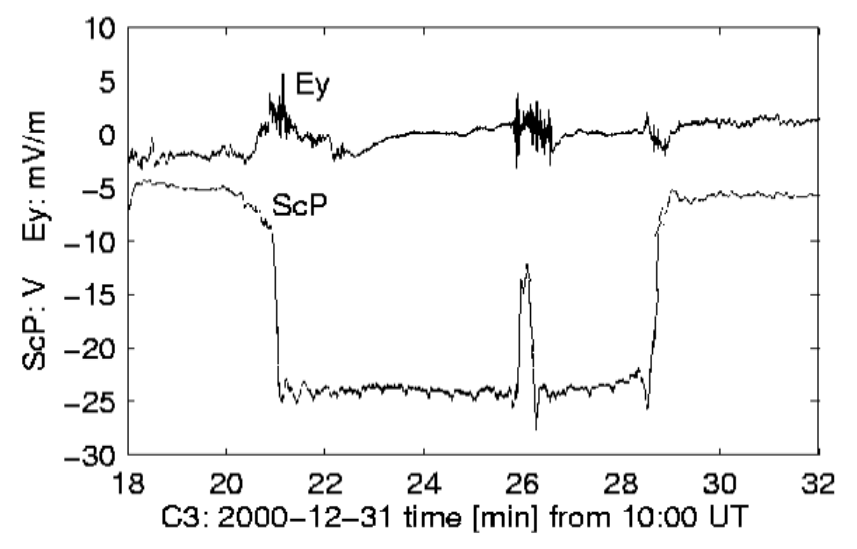

Fig. 10. Electric field component and spacecraft potential at a sequence of magnetopause crossings. Strong electric field turbulence is co-located with the boundary crossings (density gradients).

in the magnetosheath.

In these regions, the proton gyrofrequency and the lower hybrid frequency are about 0.3 and $10 \mathrm{~Hz}$, respectively. Currents at the magnetopause, density gradients, and shear in the quasi-static electric field (Sibeck et al., 1999, and references therein) can cause waves in this frequency range. An important question is how much of the broadband emissions in the satellite frame is due to true time variations, and how much is caused by a Doppler shift due to the moving magnetopause. Detailed studies (see André et al., 2001, this issue) indicate that in the magnetopause frame, both are essentially static, and also time varying. In addition, electric field structures can be present. Future investigations will include estimates of the wave polarization using single satellites, and tests of the coherence of signals between satellites, to study the generation, the frequency in the frame of the moving magnetopause, and the importance for transport across the magnetopause of these waves.

\subsection{Kinetic Alfvén and drift waves at the magnetopause}

Low-frequency turbulence extending from well below the ion gyrofrequency $f_{c i}$ up to the lower hybrid frequency $f_{l h}$ is of considerable interest for space plasmas since it contains a large wave power, but its nature and origin is not well understood. The ELF turbulence is observed by the satellites in all active regions of the magnetosphere, and in particular, at the magnetopause layer, where it is expected to play an important role in the processes of reconnection, diffusion, mass and energy transport. Earlier studies of broadband turbulence measured in the auroral zone by Freja (Stasiewicz et al., 2000) and in the high-latitude boundary layer by Polar (Stasiewicz et al., 2001) indicate that this turbulence consists of short spatial scale dispersive Alfvén waves, which are Doppler shifted in the satellite frame by plasma motion with respect to the satellite. Similar analysis has been carried out on Cluster data for an event from multiple boundary crossings. During a single passage on 31

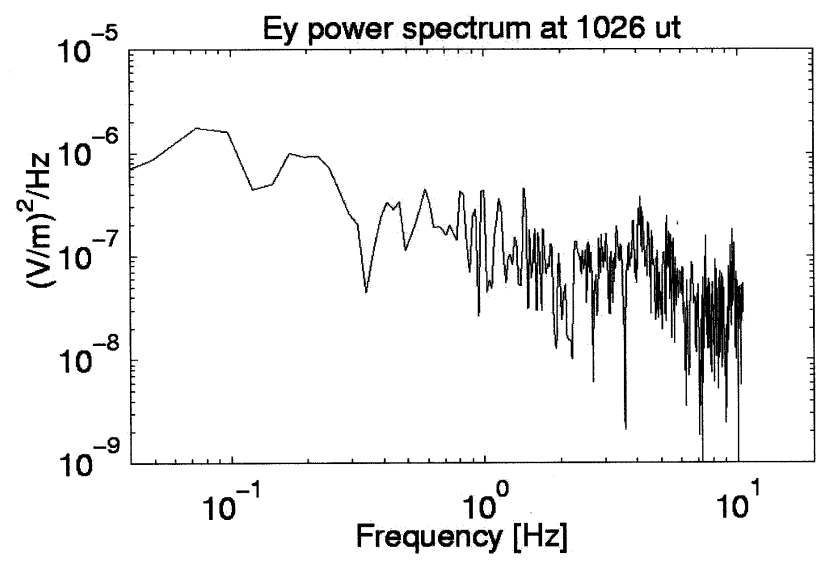

Fig. 11. Electric field power at time 10:26 UT.

December 2000, 120 magnetopause crossings were recorded during several hours, from 09:00-16:00 UT. These magnetopause crossings are characterized by strong density gradients where the density increases from a magnetospheric value of $0.1 \mathrm{~cm}^{-3}$ to about $10 \mathrm{~cm}^{-3}$ in the magnetosheath over a distance of $500-1000 \mathrm{~km}$. An example is shown in Fig. 10. The curve (scp) with the potential shows that the spacecraft was in a region with high density plasma (magnetosheath) when an outward boundary motion moved the satellite into the magnetosphere and then back toward the magnetosheath at 10:29 UT. The structure at 10:26 UT corresponds to a partial inward/outward boundary motion. Using data from the four spacecraft we can estimate the boundary speed to be $170 \mathrm{~km} / \mathrm{s}$ and the thickness of the density ramp to be $1000 \mathrm{~km}$ for the first crossing. The electric field in Fig. 10 exhibits strong turbulence during the boundary crossings. Turbulent fields of $5 \mathrm{mV} / \mathrm{m}$ are larger than the DC field of $2 \mathrm{mV} / \mathrm{m}$, and are clearly co-located within the density gradients. The power spectrum of the electric field measured around 10:26 UT is shown in Fig. 11. The electric field spectrum shows a power-law dependence.

A preliminary analysis of electromagnetic fluctuations observed by Cluster at the magnetopause layer shows that waves in the frequency range $\sim 0.03-0.3 \mathrm{~Hz}$, ie. below the proton gyrofrequency $\left(f_{c i} \approx 0.3 \mathrm{~Hz}\right.$ in the analyzed case) could be identified as kinetic Alfvén waves, which appear to have a wide spectrum of spatial scales: from large-scale wavelengths which can be resolved by four Cluster spacecraft, to structures smaller than an ion gyroradius. The existence of small wavelengths is inferred from a theoretical polarization relation which is found to agree with Cluster measurements. Small-scale structures are Doppler shifted by fast convective flows and observed as higher frequency waves in satellite data (in our case, between the ion gyrofrequency and the lower hybrid frequency; $0.3-10 \mathrm{~Hz}$ ). The results corroborate earlier findings made with Freja at ionospheric altitudes (Stasiewicz et al., 2000), and POLAR at the high-latitude boundary layer (Stasiewicz et al., 2001), that widespread ELF turbulence can be interpreted as Doppler 
Cluster sc/1
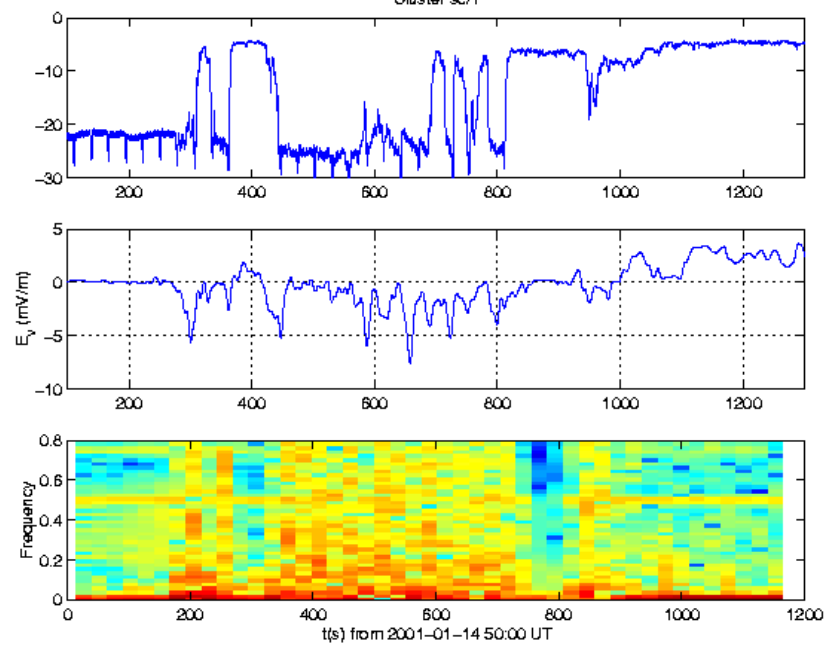

Fig. 12. Spacecraft potential and electric field data. Top panel shows the negative of the satellite potential. The middle panel shows the radial component of the electric field which has been lowpass filtered at $0.1 \mathrm{~Hz}$. There is a data gap around 15:05 UT. The bottom panel shows the spectrogram of the non-filtered electric field.

shifted dispersive Alfvén waves at small perpendicular wavelengths. Further studies are needed to assess the generation mechanism of these waves and structures, and their role in the process of energy/mass transport and diffusion across the magnetosphere.

7.4 Example of magnetopause surface wave with a period of several tens of seconds

Here we show an example where Cluster satellites observe multiple magnetopause crossings with periods of several tens of seconds ( $\mathrm{Pc} 3$ time scale). The magnetopause crossings were at 14:50-15:20 UT, on 14 January 2001 in the early afternoon sector $(\sim 15 \mathrm{MLT})$. Using data from all 4 spacecraft we show that multiple crossings are caused by an antisunward propagating surface wave. We estimate the characteristic spatial scales of the wave and the magnetopause itself. In terms of the magnetopause, here we mean the region where the plasma changes its character from magnetospheric to magnetosheath-like, which is seen as a jump in the satellite potential. This does not necessarily agree with a magnetopause current region.

Figure 12 shows an overview of the magnetopause crossing as seen by one spacecraft. The top panel shows the negative of the spacecraft potential; we can distinguish regions of magnetospheric plasma (large negative values) and magnetosheath plasma (small negative values). The sharp transition between the two indicates that the magnetospheric plasma is most likely on the closed field lines. Data from ion and electron instruments confirm this (not shown). The middle panel shows the radial component of the electric field (positive is outwards). We can see that the lowest electric field values are within the magnetosphere, but not in the direct neighbour-
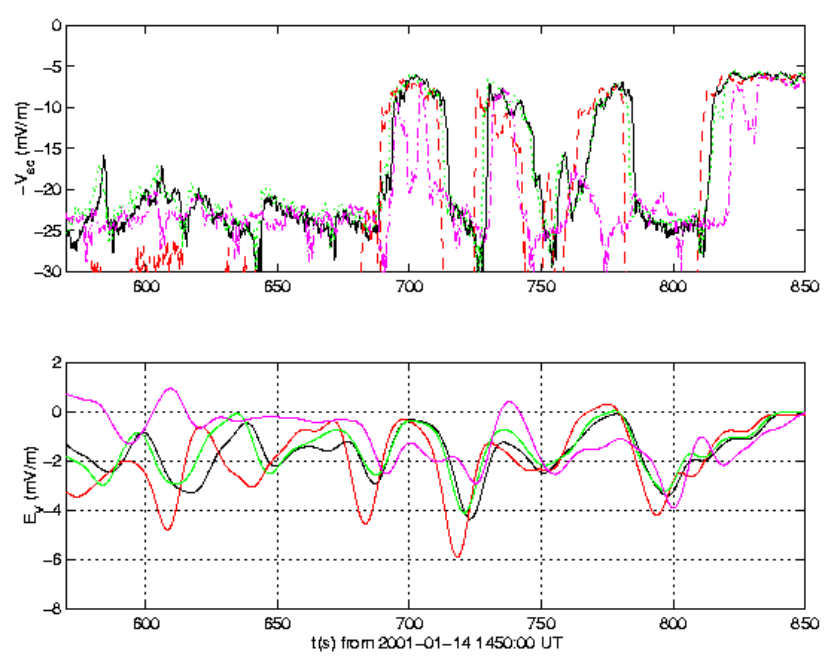

Fig. 13. For all four spacecraft, potential and the radial component of electric field, lowpass filtered at $0.06 \mathrm{~Hz}$.

hood of the magnetopause (jump in the spacecraft potential). However, near the magnetopause crossing, we see large negative values of the electric field inside the magnetosphere. The corresponding $\boldsymbol{E} \times \boldsymbol{B}$ velocity is of the order of $200 \mathrm{~km} / \mathrm{s}$ anti-sunward that is comparable to the plasma velocity in the magnetosheath. Similar electric fields has been seen by Geotail (Mozer et al., 1994). The bottom panel shows the electric field spectrogram where we can see increased wave activity at the magnetopause in the frequency range both above and below the proton gyrofrequency (it is between 0.2 and 0.4 Hz). From the top panel of Fig. 12 around 15:03 UT, we can identify four magnetopause crossings with a period of about 30-40 s. The same crossings can be identified as oscillations in the electric field. However, the electric field data show several additional oscillations of the same period before crossings can be seen in the satellite potential, i.e. during this period the electric field can be used as the remote sensor of the magnetopause disturbances. We will study closer the magnetopause crossings that are clearly seen in the satellite potential.

Figure 13 shows the satellite potential and the radial electric field component from all spacecraft. Not all magnetopause crossings are observed by all 4 spacecraft. Thus, the third back-and-forth crossing is seen only by spacecraft 1,2 and 3, while during the first and last crossings, spacecraft 4 makes short excursions back into the magnetosphere. This can be understood if we take into account the fact that spacecraft 1,2, and 3 lie approximately in the plane of the undisturbed magnetopause, while spacecraft 4 is located about $400 \mathrm{~km}$ inwards. But this also tells us that the amplitude of magnetopause oscillations perpendicular to the magnetopause is only slightly larger than the $400 \mathrm{~km}$ distance between spacecraft 4 and the other spacecraft. Similar reasoning allows us to estimate the thickness of the region where we see large electric fields inside the magnetosphere to be about $500 \mathrm{~km}$. This is comparable to the gyroradius of the 

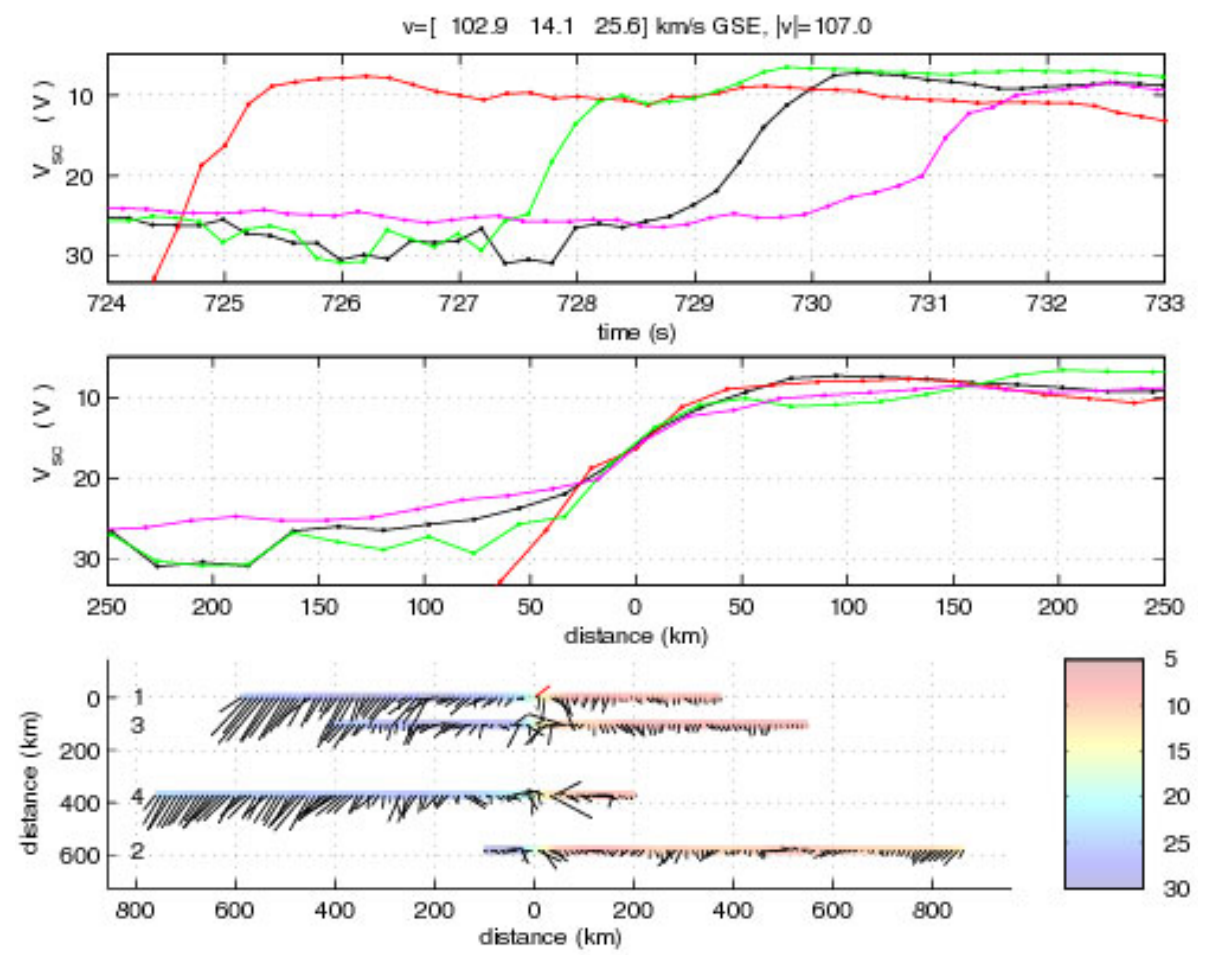

Fig. 14. Top panel shows the satellite potential of all four spacecraft for an outbound magnetopause crossing at 15:02:10 UT, 14 January 2001. During this day, the EDI instrument on spacecraft 2 significantly affected the spacecraft potential in low density plasma ( $-V s c$ below about $-20 \mathrm{~V}$ ). The middle panel shows the potential structure as a function of distance. The velocity has been obtained from the time when the spacecraft potentials cross $-15 V$ value. The obtained velocity in GSE coordinates is $v=(-103,14,26) \mathrm{km} / \mathrm{s}$. The bottom panel is a combined plot: $X$-axis is in the direction antiparallel to the velocity of the magnetopause, $Y$-axis is perpendicular to $X$ and the magnetic field (thus in the plane of the magnetopause). The satellite trajectory is projected onto this plane and colored according to $-V s c$ values. In addition, the black lines show the direction of the electric field.

plasma sheet ion with a characteristic energy of $4 \mathrm{keV}$.

To proceed with phase velocity estimates, we have to look in more detail at separate magnetopause crossings. In Fig. 14, we zoom into one outbound magnetopause crossing at about 15:02:10 UT. The top panel shows that most of the change in the satellite potential occurs within a time period of less than a second. The spatial scale is shown in the middle panel. It is obtained by estimating the velocity of the magnetopause along its normal direction, which in this case, is $107 \mathrm{~km} / \mathrm{s}$, from the times at which $-V s c$ at all 4 spacecraft pass the $15 \mathrm{~V}$ level. The assumption is made that the magnetopause on the scale of satellite separation is flat and moves with a constant velocity. We can see that most of the satellite potential change is within a distance of less than $100 \mathrm{~km}$. This is comparable to the gyroradius of $100 \mathrm{eV}$ magnetosheath protons, which is about $70 \mathrm{~km}$. In the bottom panel, we show the electric field data around the magnetopause crossing in the reference frame moving with the magnetopause (without $\boldsymbol{V} \times \boldsymbol{B}$ correction). The electric field has been calculated assuming that its component parallel to $B$ is zero. One can see the large electric fields inside the magnetosphere which have both tangential and normal components with respect to the magnetopause. During this crossing, the field becomes small approximately $100 \mathrm{~km}$ before the mag- netopause; however, during other crossings, the field can be large until the magnetopause itself is reached. At the magnetopause itself, the field is strong but very variable.

From the difference in magnetopause velocity directions for the inbound and outbound crossings we can see that the multiple crossings, are consistent with surface waves that propagate anti-sunward along the magnetopause. Therefore, we would like to observe the wave structure in the reference frame that moves with the phase velocity of this surface wave. We estimate the direction and size of the surface wave phase velocity such that it is in the plane defined by the inbound and outbound crossings and is consistent with both of them. Here, for simplicity, we take only two crossings (at $715 \mathrm{~s}$ and $730 \mathrm{~s}$ ). The obtained phase velocity is about $190 \mathrm{~km} / \mathrm{s}$ anti-sunward along the magnetopause, $v=(-144,79,89) \mathrm{km} / \mathrm{s}$ in GSE, and is comparable to the magnetosheath plasma velocity. We can compare the angles between this phase velocity and the magnetopause normals at the outbound $\left(55^{\circ}\right)$ and inbound $\left(69^{\circ}\right)$ crossings. We see that the outbound crossing is slightly steeper.

In Fig. 15, we plot a combined plot, in the same way as in the bottom panel of Fig. 14, to show the electric field and potential in the frame of reference of the surface wave ( $E$ field is without $\boldsymbol{V} \times \boldsymbol{B}$ correction). 


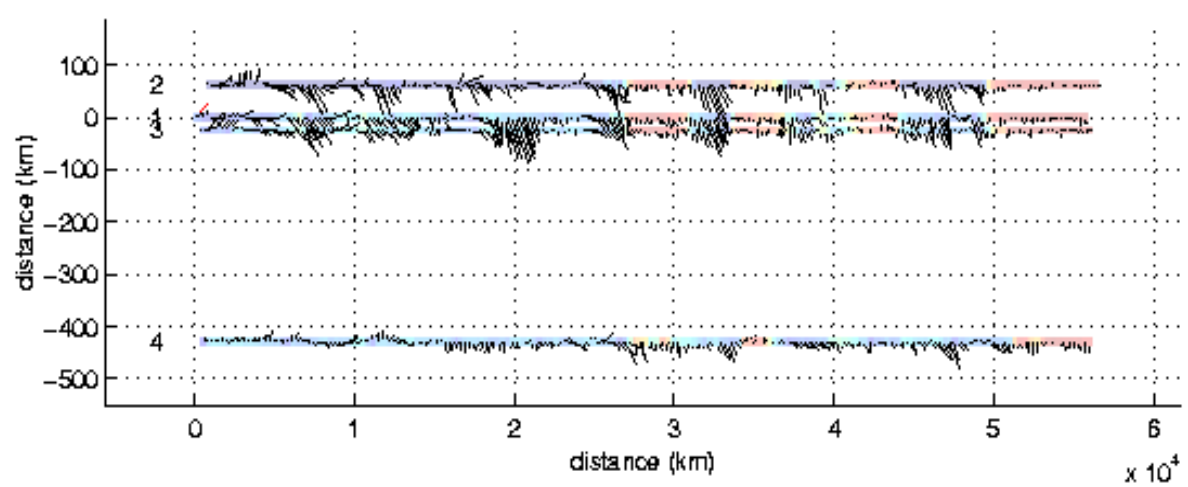

Fig. 15. A combined plot: $X$-axis is in the direction antiparallel to the phase velocity, $v=(-144,79,89) \mathrm{km} / \mathrm{s}$ GSE, of the surface wave along the magnetopause, $Y$-axis is perpendicular to $X$ and the magnetic field (thus in the plane of the magnetopause). The satellite trajectory is projected onto this plane and colored according to $-V s c$ values. In addition, the black lines show the direction of the electric field.

Spacecraft 1, 2 and 3 project close to each other since the plane defined by them is close to being parallel with the magnetopause plane. One should note the different scaling on the axis. The wavelength of the surface wave along the magnetopause is of the order of $6000 \mathrm{~km}$. In the regions of the magnetopause crossings the electric field inside the magnetosphere is almost perpendicular to the phase velocity of the surface wave without large variations in the electric field direction. Thus, the electric field does not follow large variations in the normal direction of local magnetopause crossings (as we see from above, this variation can be more than $60^{\circ}$ ) but tends to agree with the normal direction of the "mean" (undisturbed) magnetopause.

Further studies of this and similar magnetopause crossings should address the questions of whether these surface waves with periods of several tens of seconds are driven by the solar wind or generated through Kelvin-Helmholtz type of instabilities. What is the source of the large electric field just inside the magnetopause and does it map to the ionosphere? Does this electric field contribute to the convection potential drop?

\subsection{Solar wind and magnetosheath flows}

An example is given of a period of more than three hours, during which the Cluster spacecraft were in the magnetosheath and solar wind (Fig. 16). The data from all four spacecraft are similar for this time interval. For simplicity, only the data from spacecraft 4 is illustrated. During the plotted time interval, the ACE spacecraft was in the upstream solar wind, where it measured an average plasma density of about 6 particles $/ \mathrm{cm}^{3}$ and a flow speed of $310 \mathrm{~km} / \mathrm{s}$. At about 19:35 UT, ACE observed an abrupt northward turning of the interplanetary magnetic field, which arrived at Cluster near 21:00 UT to modify the fields as discussed below.

The top graph is the spacecraft potential, which is a function of plasma density. It shows that spacecraft 4 was in the magnetosheath between about 19:49:30-20:03:30 UT and again between about 21:45:30-22:40:00 UT. During the re- mainder of the figure, spacecraft 4 was in the solar wind, where the average spacecraft potential of about 7 volts corresponds to an average density of about 7 particles $/ \mathrm{cm}^{3}$ (which compares to the average ACE density of about 6 particles/cm3).

The second and third panels give the spin period averaged electric field components in the GSE $X$ - and $Y$-directions. The field was turbulent in the magnetosheath and relatively quiet in the solar wind. The central feature of this electric field data is that $E_{X}$ and $E_{Y}$ were correlated, as they generally are in the solar wind and magnetosheath. This correlation can be understood by assuming that the total flow velocity in the GSE- $Z$ direction, $\mathrm{VTOT}_{Z}$, is 0 (as it generally is in the magnetosheath and solar wind). Then,

$$
V T O T_{Z}=0=v_{\|}\left(B_{Z} / B\right)+\left(E_{X} B_{Y}-E_{Y} B_{X}\right) / B^{2}
$$

This equation may be viewed as giving the parallel flow, $\mathrm{v}_{\|}$, as a function of the field quantities. Substituting this function into expressions for $\mathrm{VTOT}_{X}$ and $\mathrm{VTOT}_{Y}$ gives, after setting the parallel electric field to zero,

$V T O T_{X}=E_{Y} / B_{Z}$

$V T O T_{Y}=-E_{X} / B_{Z}$

These two equations show that $E_{X}, E_{Y}$, and $B_{Z}$ correlate with each other. Thus, the correlation of the electric field components is a consequence of the total flow being approximately constant and confined to the ecliptic plane. A further consequence of this correlation is that the total flow (not just the $\boldsymbol{E} \times \boldsymbol{B}$ component of the flow) is obtained from the ratios of field quantities given in Eqs. (9) and (10) and that are plotted in the lowest two panels of Fig. 16.

Several conclusions may be reached from the lowest two plots:

1. The average flow observed by Cluster in the solar wind was $298 \mathrm{~km} / \mathrm{s}$. This can be compared to the average flow of $310 \mathrm{~km} / \mathrm{s}$ observed by ACE. (It is pointed out that the ACE data are preliminary). 


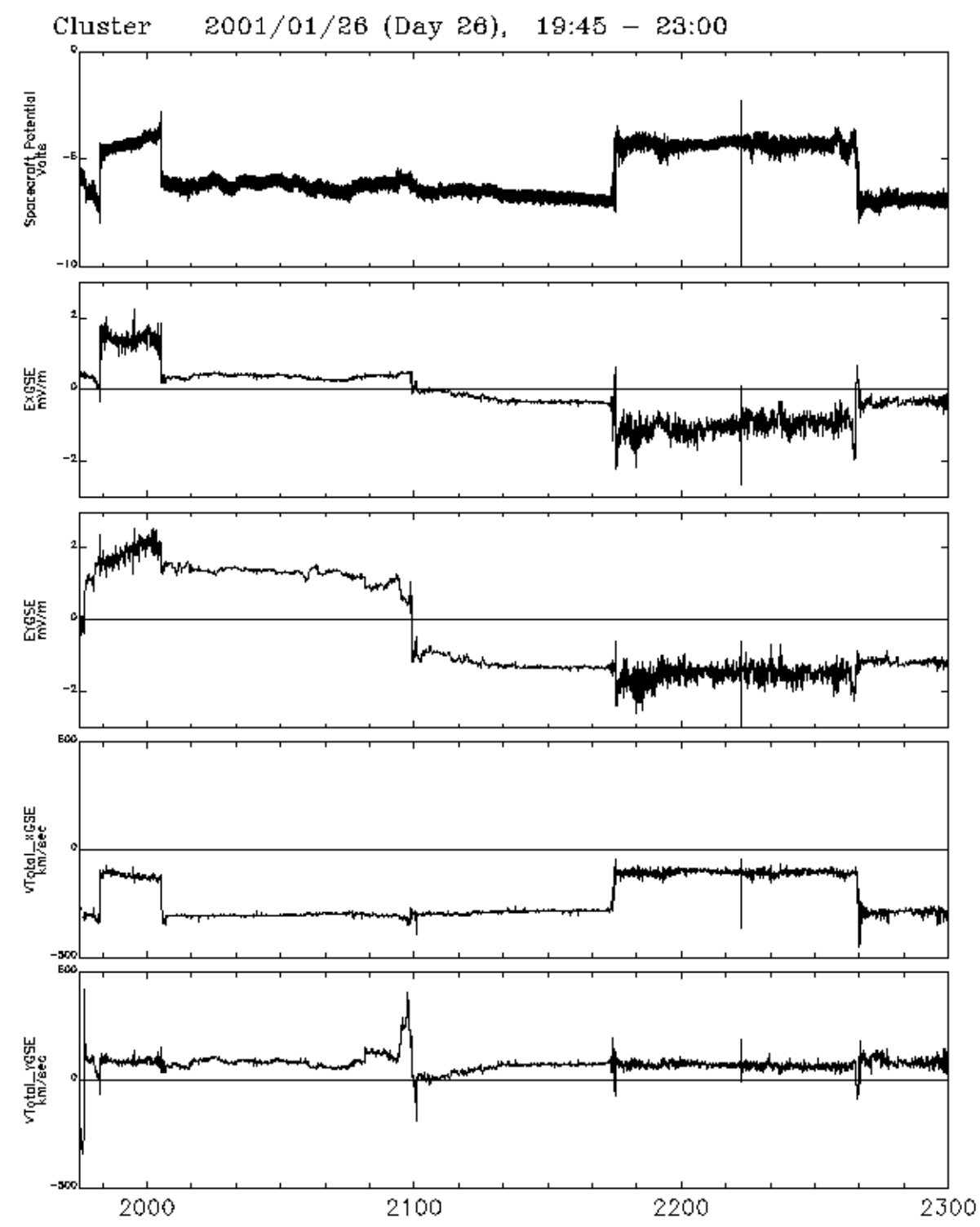

Fig. 16. Example of flow velocities for a period of more than three hours, during which the Cluster spacecraft were in the magnetosheath and solar wind.

2. In the magnetosheath, the $X$-component of the flow is reduced by a factor of about 4 relative to that in the solar wind. The magnetosheath flow appears much less turbulent than the fields from which it was obtained.

3. In the solar wind, near 21:00, the electric fields changed sign but the solar wind flow velocity did not. This is proof that the calibration offsets in the field measurements are correct.

4. The average $Y$-component of the flow in the solar wind was about $+60 \mathrm{~km} / \mathrm{s}$, which is consistent with the expectations associated with the aberration angle of the solar wind flow.

This study shows that the electric field, its offsets, and the spacecraft potential have been calibrated to an absolute accuracy of better than $10 \%$ in this and several similar examples.
The $Z$-component of the magnetic field has been similarly calibrated.

\section{Turbulent boundary layer}

We would like to illustrate the new Cluster electric field data with an example from one of the most interesting critical magnetospheric regions: turbulent boundary layer (TBL; see e.g. Savin et al., 1998) at the cusp/magnetosheath interface. Both the orbit and electric field data quality permit for the first time the exploration of statistical features of the electric field turbulence in the region of developed non-linear turbulence with the signatures of self-organization and multi-scale cascading. The magnetic turbulence signatures in TBL are 


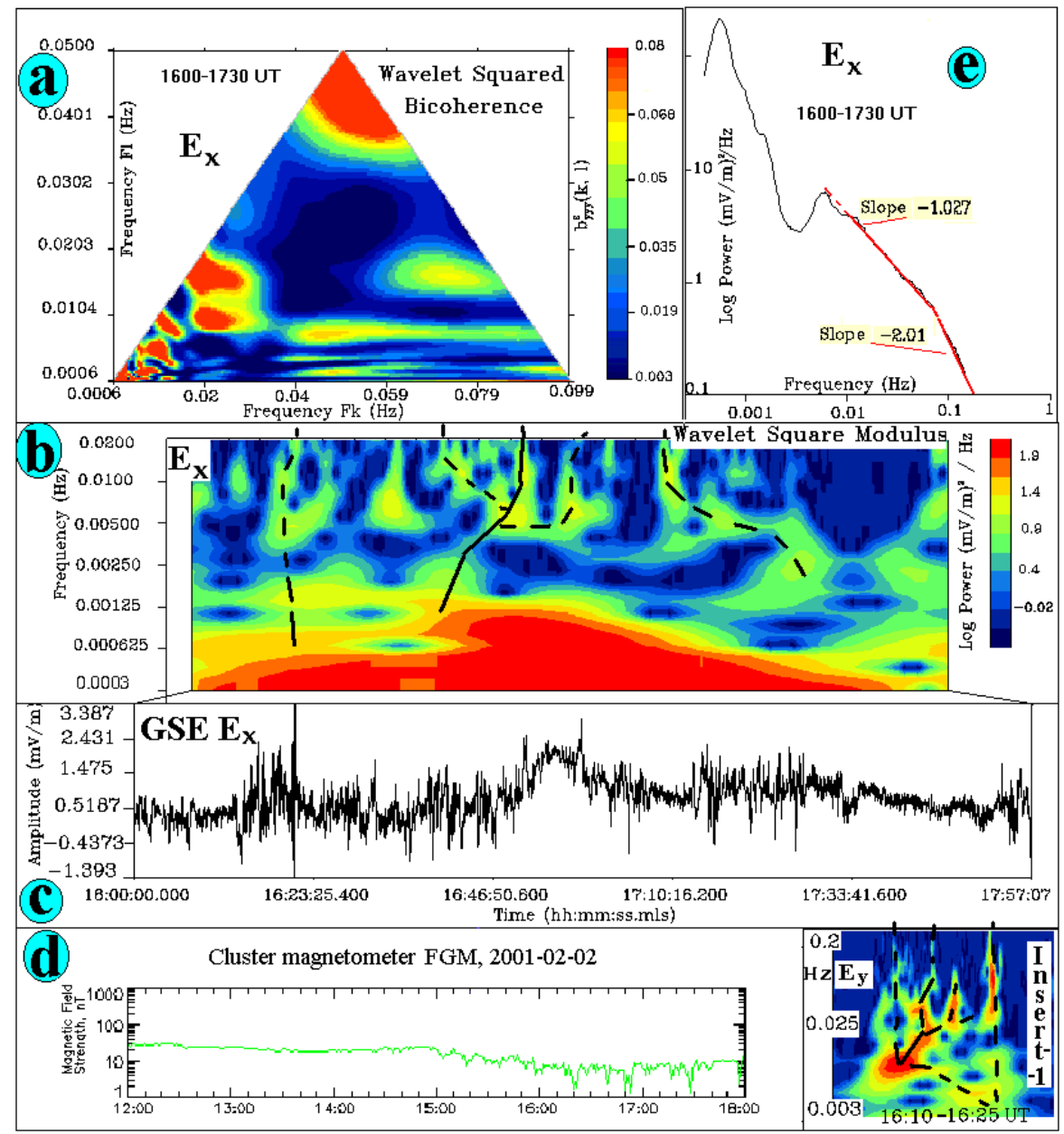

Fig. 17. An outbound turbulent boundary layer crossing by Cluster on 2 February 2001. Panels (a) and (e) show $E_{X}$ bi-spectrogram and integrated spectrum at 16:00-17:30 UT, respectively. Panel (b) displays a wavelet $E_{X}$ spectrogram in the range of 3-20 mHz, inferred cascades are depicted by black lines. Panel (c) demonstrates the electric field $E_{X}$ waveform and panel (d) the magnetic field. Note, in particular, that the magnetic field drops to very low values in a few cases. Insert 1 in panel (d) shows the most characteristic example of the cascade-like $E_{Y}$ wavelet spectrogram at 16:10-16:25 UT (see text for details).

described in Savin et al. (2001) (see there also the respective references).

In Fig. 17, we depict an outbound TBL crossing of the Cluster spacecraft 1 at high latitudes. In panel (d), the magnetic field $|\boldsymbol{B}|$ summary plot illustrates a very common TBL signature of "diamagnetic bubbles" with magnetic field drops down to a few nT. We have studied the spacecraft potential behaviour that reflects plasma density fluctuations induced by the "diamagnetic bubbles". While having generally the same character as the electric field components, these fluctuations have no such characteristic peculiarities as the magnetic field components described by Savin et al. (2001). We think this is due to the strong dominance of the transverse waves in the TBL while the density variations are connected with the compressible fluctuations.

In panel (c) of Fig. 17, we depict the $E_{X}$ waveform in the GSE frame. One can see that the general electric field activity tends to correlate with the $|\boldsymbol{B}|$ gradients in the panel (d).
Panel (b) displays a wavelet colour-coded spectrogram of $E_{X}$ in the frequency band $0.3-20 \mathrm{mHz}$. Panels (a) and (e) represent a wavelet bi-spectrogram and wavelet integrated spectrum, respectively, in the bands $0.6-99$ and $0.35-170 \mathrm{mHz}$ (see the wavelet description below).

We start from the panel (b) description. Wavelet analysis is a powerful tool to investigate turbulent signals and shortlived structures. In order to examine the transient non-linear signal in the TBL, we have performed the wavelet transform with the Morlett wavelet:

$$
\begin{aligned}
& W(a, t)=C \sum\left\{f\left(t_{i}\right)\right. \\
& \left.\quad \exp \left[i 2 \pi\left(t_{i}-t\right) / a-\left(t_{i}-t\right)^{2} / 2 a^{2}\right]\right\}
\end{aligned}
$$

where " $C$ " has been chosen so that the wavelet transform amplitude $|W(a, t)|$ is equal to the Fourier. This wavelet shows the single peak at the frequency $f=1 / a$, with a wavelet characteristic scale representing a frequency $f=1 / a \pm f / 8$. 
We use SWAN software from LPCE/CNRS in Orleans for the wavelet analysis. The wavelet spectrograms in panel (b) clearly outline wave-trains (i.e. isolated maximums) at different frequencies simultaneously, which indicates multiscale processes. The linkage between the maximums can represent a feature of cascade processes; the direct/inverse (i.e. high or low frequency fluctuations appear first) cascades could be recognized. We mark the probable cascades by black lines; cascade-like features could be recognized, developing almost simultaneously or developing over time. The most prominent cascade-like $E_{Y}$ spectrogram is blown up in Insert 1 of panel (d). A disturbance at $\sim 10 \mathrm{mHz}$, is accompanied by the lower intensity spectral maximums up to $200 \mathrm{mHz}$ and then followed by intensive wave-trains at higher and higher frequencies. The lower intensity branch descends to a few $\mathrm{mHz}$ resembling a reverse cascade. Another reverse cascade (instant) is depicted on the right part of Insert 1. In panel (b), we would like to outline the processes at about $0.4-2$ and 5-9 $\mathrm{mHz}$, where the maximums near the two lowest frequencies are visible throughout the TBL, i.e. the region of the "diamagnetic bubbles" in panel (d). Their junction and intensification seem to trigger a direct cascade. Both continuous and spiky (i.e. low and high frequency) activities in panel (b) correspond to maximums in the integrated spectrum in panel (e), (note that the latter was calculated for 16:00-17:30 UT). In the high frequency disturbances on panel (e) two characteristic slopes are seen: -1.027 at $0.006-0.08 \mathrm{~Hz}$ and -2.01 at $0.08-0.2 \mathrm{~Hz}$. The slope at the higher frequency corresponds to the self-consistent plasma turbulence and is similar to the one observed in the magnetotail. The lower frequency part may be related to the "flicker" noise and is also a general feature of non-linear systems near a state of self-organized criticality. The transient wave-trains, visible in panel (b) at $5-10 \mathrm{mHz}$ should provide the mean for the energy transfer towards the higher frequencies, in contrast to the chaotic quasi-homogeneous processes at higher frequencies. The electric field spectral slopes remarkably resemble that of the magnetic spectra in the TBL from Polar and Interball-1 data (Savin et al., 2001).

We would like to do a further test for possible selforganization in the TBL processes. We use the wavelet-based bicoherence to check if the wave-trains at different frequencies really constitute the coherent interactive system with multi-scale features. In the SWAN software, for the wavelet analysis, the bicoherence is defined as:

$$
\begin{gathered}
b^{2}\left(a_{1}, a_{2}\right)=\left|B\left(a_{1}, a_{2}\right)\right|^{2} /\left\{\sum \mid W\left(a_{1}, t_{i}\right)\right. \\
\left.\left.W\left(a_{2}, t_{i}\right)\right|^{2} \sum\left|W\left(a, t_{i}\right)\right|^{2}\right\}
\end{gathered}
$$

with $B\left(a_{1}, a_{2}\right)$ representing the normalized squared wavelet bi-spectrum:

$$
B\left(a_{1}, a_{2}\right)=\sum W^{*}\left(a, t_{i}\right) W\left(a_{1}, t_{i}\right) W\left(a_{2}, t_{i}\right)
$$

the $W(a, t)$ is the wavelet transform according to (11) and the sum is performed, satisfying the following rule:

$$
1 / a=1 / a_{1}+1 / a_{2}
$$

which corresponds to a frequency sum rule for the 3-wave process, $f=f_{1}+f_{2}$. The bicoherence has substantial value if three processes, with the highest frequency being the sum of the other two, are phase-coupled. The simplest such case is the harmonic generation due to quadratic non-linearity, namely the second harmonic generation with $2 f=f+f$, the third harmonic one with $3 f=2 f+f$, etc. The harmonics are present in any non-linear wave pulse and do not represent phase coupling of the different waves; one can see them at the right border of panel (a). We assume that the dominating non-linear process in the TBL is a 3-wave decay (or junction). This is the most powerful non-linear wave process (excluding the harmonics) which requires the thirdorder non-linearity in the system. We will not discuss here the weaker higher-order non-linear effects, which might also contribute to the TBL physics.

In panel (a) of Fig. 17, we present a bicoherence spectrogram for GSE $E_{X}$ in the Cluster outbound TBL. The frequency plane $\left(f_{1}, f_{2}\right)$ is limited by the signal symmetry considerations and by the frequency interval of the most characteristic TBL slope of $\sim 1$ in panel (e). As we mentioned in the previous paragraph, the bicoherence in the TBL displays the second and higher harmonic non-linear generations and, most likely, the 3-wave processes. We assume cascade-like signatures in Fig. 17 when the bicoherence at the sum frequency, $f=f_{1}+f_{2}$, has comparable a value with that of point $\left(f_{1}, f_{2}\right)$. In the case of the horizontal-spread maximum, it implies that the wave at sum frequency interacts with the same wave at the initial frequency (say, $f_{A}$ ) in the following 3-wave process: $f_{3}=f_{A}+f$, etc.; the initial wave spectrum can be smooth, resulting in the continuous bi-spectral maximum. One can see the most characteristic picture of the synchronized 3-wave processes in a rather wide frequency band in the horizontalspread maximum at $\sim 6-9 \mathrm{mHz}$ in panel (a). It corresponds to a maximum and plateau on panel (e) and to the most intensive wave-trains in panel (b) at 16:10 17:06 UT with the cascade-like features (see also Insert 1 and the discussion above). A maximum that is not continuous at $\sim 1-2 \mathrm{mHz}$ (maximum at $\sim 16: 10-17: 00$ UT in panel (b)). A horizontal maximum is also present at $\sim 0.6 \mathrm{mHz}$ at the lowest frequency in panel (a) (while it is quite narrow in panel (a), we have checked that it is present on the lower frequency bi-spectrograms; see also panels (b) and (e)). A weak horizontal maximum in panel (a) at $3.5 \mathrm{mHz}$ could correspond to a cascade-like event in panel (b) at $\sim 17: 13$ UT. Numerous wave-trains in panel (b) at $15-19 \mathrm{mHz}$ can contribute to the corresponding horizontal maximum in panel (a). Most of these maximums are also present in the spacecraft potential bi-spectrograms (not shown), with the most prominent ones are at $2-3$ and $0.6 \mathrm{mHz}$.

Thus, the electric field TBL disturbances have specific slopes and other features, and the bi-spectral phase coupling indicates a self-organization of the TBL as the entire region. Inverse cascades of the kinetic Alfvén waves are proposed to form the larger scale resonant structures, which, in turn, synchronize all non-linear direct cascades in the TBL in a selfconsistent manner (Savin et al., 2001). Lower frequency (few 
$\mathrm{mHz}$ ) fluctuations might resonate with the cusp flux tubes and provide a possible link to correlated solar wind interactions with the dayside magnetopause and cusp ionosphere.

We would like to emphasize that our consideration of the non-linear cascade scenario, while in agreement with the Interball-1 and Polar case studies, is a very likely hypothesis, which could be further checked using multi-spacecraft data at least for the lower frequencies. In addition, verification of wave vector sums conservation in 3(4)-wave nonlinear processes, wave mode identifications, group velocity determinations, data transformations into de Hoffman-Teller or magnetopause frames, etc. are foreseen the verification of our hypothesis. For that study plasma moments and magnetic fields at the highest time resolution will be needed.

\section{Summary}

The coherence between observations of the electric field from the four Cluster spacecraft is very high, for frequencies below the spin period for several regions along the orbit. This preliminary study shows cross-correlation values of 0.9 or higher for the low frequencies, which indicates that true three-dimensional investigations can be carried out for many plasma phenomena. Wave spectra up to several $\mathrm{kHz}$ also show similarities that look encouraging for future detailed comparisons between spacecraft. It is also demonstrated that the spacecraft potential, a measure of the plasma density, is very useful for the identification and characterization of rapidly moving boundaries near the magnetopause, which is one of the primary regions for Cluster studies. The potential of four point-measurements are used, in particular, to evaluate surface waves with wavelengths of about $6000 \mathrm{~km}$ along the magnetopause. In the auroral oval region, the four satellite observations give important information on electric field structures. The preliminary study presented here indicates that the electric field structure on auroral field lines retained its major features for more than three minutes. This suggests that it represents the upward continuation of a quasi-static Ushaped potential contour associated with an auroral arc. The persistency of the structure provides support for a quasi-static auroral potential structure that extends at least to a geocentric distance of $4.3 R_{E}$.

The large potential for three-dimensional investigations in several regions along the orbit is demonstrated and it may be expected that studies based not only on electric field data but also on observation from the full complement of instruments on board Cluster will open the way for great scientific discoveries.

Acknowledgements. Work on turbulent boundary layer was partially supported by the grant INTAS 97-1612. We appreciate D. Lagoutte and his colleagues from LPCE/CNRS in Orleans for providing the SWAN software used for the wavelet transform analysis. Magnetic field data from the FGM instrument (A. Balogh), electron density data from the Whisper instrument (P. Décréau) and search coil magnetic field data from the STAFF instrument (N. CornilleauWehrlin) used in this study is greatly acknowledged.
Topical Editor M. Lester thanks H. Alleyne and P. Stauning for their help in evaluating this paper.

\section{References}

André, M., Behlke, R., Wahlund, J.-E., et al.: Multi-spacecraft observations of broadband waves near the lower hybrid frequency at the Earthward edge of the magnetopause, Ann. Geophysicae, this issue, 2001.

Balogh, A., Dunlop, M.W., Cowley, W.H., et al.: The Cluster magnetic field investigation, Space Sci. Rev., 79, 65-92, 1997.

Carpenter, D. L. and Lemaire, J.: Erosion and recovery of the plasmasphere in the plasmapause region, Space Sci. Rev., 80, 153 179, 1997.

Décréau P. M. E., Fergeau,, P., Krasnosels'kikh, V., et al.: Early results from the WHISPER instrument on Cluster: an overview, Ann. Geophysicae, this issue, 2001.

Escoubet, C. P., Pedersen, A., Schmidt, R., and Lindqvist, P.-A.: Density in the magnetosphere inferred from the ISEE-1 spacecraft potential, J. Geophys. Res., 102, 17, 595-609, 1997.

Gurnett, D. A., Pickett, J. S., Huff, R. L., et al.: First results from Cluster wide-band plasma wave investigation, Ann. Geophysicae, this issue, 2001.

Gustafsson, G., Bergström, R., Holback, B. et al.: The electric field and wave experiment for the Cluster mission, Space Sci. Rev., 79, 137-156, 1997.

Laakso, H. and Jarva, M.: Evolution of the plasmapause position, J. Atmos. Sol. Terr. Phys., in press, 2001.

Laakso, H., Pfaff, R., and Janhunen, P.: Polar Observations of Electron Density Distribution in the Earth's Magnetosphere, J. Geophys. Res., submitted, 2000.

Lemaire, J. F. and Gringauz, K. I.: The Earth's Plasmasphere, Cambridge Univ. Press, Cambridge, 1998.

Marklund, G., Karlsson, T., and Clemmons, J.: On low-altitude particle acceleration and intense electric fields and their relationship to black aurora, J. Geophys. Res., 102, 17 509-17 522, 1997.

Mozer, F. S., Hayakawa, H., Kokobun, S., Nakamura, M., Okada, T., Yamamoto, T., and Tsuda, K.: The morningside low-latitude boundary layer as determined from electric and magnetic field measurements on Geotail, Geophys. Res. Lett., 21, 2983, 1994.

Nakagawa, T., Ishii, T., Tsuruda, K., Hayakawa H., and Mukai, T.: Net current density of photoelectrons emitted from the surface of the Geotail spacecraft, Earth Planets Space, 52, 283-292, 2000.

Pedersen, A.: Solar wind and magnetosphere plasma diagnostics by spacecraft electrostatic potential measurements, Ann. Geophysicae, 13, 118-121, 1995.

Pedersen, A., Mozer, F., and Gustafsson, G.: Electric field measurements in a tenuous plasma with spherical probes, AGU Geophys. Monograph 103, 1-12, 1998.

Pedersen, A., Décréau P. M. E., Escoubet, C.-P., et al.: Cluster four-point high time resolution information on electron densities, Ann. Geophysicae, this issue, 2001.

Savin, S. P., Borodkova, N. L., Budnik, E. Yu., et al.: Interball tail probe measurements in outer cusp and boundary layers, in Geospace Mass and Energy Flow: Results from the International Solar-Terrestrial Physics Program, (Eds) Horwitz, J. L., Gallagher, D. L., and Peterson, W. K., Geophysical Monograph 104, AGU, Washington D.C., pp. 25-44, 1998.

Savin, S., Maynard, N., Sandahl, I., et al.: Magnetosheath/ Cusp Interface, submitted to Ann. Geophysicae, 2001. 
Scudder, J. D., Cao X., and Mozer, F.: Photoemission currentspacecraft voltage relation: Key to routine quantitative low energy plasma measurements, J. Geophys. Res., 105, 21, 281-294, 2000.

Sibeck, D. G., Paschmann, G., Treumann, R. A., Fuselier, S. A., Lennartsson, W., Lockwood, M., Lundin, R., Ogilvie, K. W., Onsager, T. G., Phan, T.-D., Roth, M., Scholer, M., Sckopke, N., Stasiewicz, K., and Yamauchi, M.: Plasma transfer processes at the magnetopause, Space Sci Rev, 88, 207, 1999.

Stasiewicz K., Khotyaintsev, Y., Berthomier, M., and Wahlund, J.E.: Identification of widespread turbulence of dispersive Alfvén waves , Geophys. Res. Lett., 27, 173, 2000.

Stasiewicz, K., Seyler, E. C., Mozer, F., Gustafsson, G., Pickett,
J., and Popielawska, B.: Magnetic bubbles and kinetic Alfvén waves in the high-latitude magnetopause boundary, J. Geophys. Res., accepted for publication, 2001.

Volland, H.: Atmospheric Electrodynamics, Springer-Verlag, Berlin, 1984.

Wooliscroft, L. J. C., Alleyne, H. St. C., Dunford, C. M., et al.: The digital wave-processing experiment for Cluster, Space Sci. Rev., 79, 209-231, 1997.

Wygant, J. R., Keiling, A., Cattell, C. A., et al.: Polar spacecraft based comparisons of intense electric fields and Poynting flux near and within the plasma sheet-tail lobe boundary to UVI images: An energy source for the aurora, J. Geophys. Res., 105, $18675-18692,2000$. 